\title{
Citrus Polyphenols in Brain Health and Disease: Current Perspectives
}

\author{
Matthew G. Pontifex, Mohammad M. A. H. Malik, Emily Connell, Michael Müller and \\ David Vauzour*
}

Norwich Medical School, Biomedical Research Centre, Faculty of Medicine and Health Sciences, University of East Anglia, Norwich, United Kingdom

In addition to essential micronutrients such as vitamin C, citrus fruits represent a considerably rich source of non-essential bioactive compounds, in particular flavanones which form a sub-set of the flavonoid group. Preclinical studies have demonstrated the neuroprotective potential of citrus flavonoids and have highlighted both the wellestablished (anti-inflammatory and anti-oxidative properties), and newly emerging (influence upon blood-brain barrier function/integrity) mechanistic actions by which these neurological effects are mediated. Encouragingly, results from human studies, although limited in number, appear to support this preclinical basis, with improvements

OPEN ACCESS

Edited by: Vittorio Calabrese, University of Catania, Italy

Reviewed by: Nadia Ferlazzo, University of Messina, Italy

Gioacchino Calapai, University of Messina, Italy

*Correspondence: David Vauzour

D.Vauzour@uea.ac.uk

Specialty section:

This article was submitted to Neuroenergetics, Nutrition and Brain

Health,

a section of the journal

Frontiers in Neuroscience

Received: 11 December 2020

Accepted: 25 January 2021

Published: 19 February 2021

Citation:

Pontifex MG, Malik MMAH Connell $E$, Müller $M$ and Vauzour $D$ (2021) Citrus Polyphenols in Brain Health and Disease: Current Perspectives.

Front. Neurosci. 15:640648. doi: 10.3389/fnins.2021.640648 in cognitive performance and disease risk observed across healthy and disease states. Therefore, citrus fruits - both as whole fruit and 100\% juices - should be encouraged within the diet for their potential neurological benefit. In addition, there should be further exploration of citrus polyphenols to establish therapeutic efficacy, particularly in the context of well-designed human interventions.

\section{Keywords: flavonoids, orange juice (OJ), cognition, gut-brain axis, neuroprotection}

\section{INTRODUCTION}

A varied and balanced diet, rich in plant-derived material (fruit and vegetables), is widely regarded as optimal for maintaining overall health and wellbeing. Unfortunately, typical modern dietary preferences are increasingly skewed in favor of nutrient poor, calorie dense, ultra-processed food choices. Combined with the background of an aging population, circumstances have produced a 'perfect storm' leading to a surge in metabolic disease burden, including rising incidence of neurodegenerative conditions which present significant societal and economic challenges.

Plant bioactives such as polyphenolic compounds have shown potential in negating the metabolic disease process (Chiva-Blanch and Badimon, 2017). Polyphenol describes a broad range of non-essential, usually naturally occurring compounds of which fruits and vegetables represent a significant source. The use of polyphenolic compounds in a therapeutic capacity is being increasingly considered, as we 'return to nature' to find sources of potential drug candidates (Xiao, 2017; Bellavite and Donzelli, 2020). Although currently lacking confirmation through rigorous long-term randomized control trial (RCT) evidence, the use of polyphenolic compounds in the prevention of cardiovascular disease appears promising (Parmenter et al., 2020). Indeed, an inverse relationship between polyphenolic compound consumption and cardiovascular disease mortality, risk and biomarkers are consistently highlighted in observational and preclinical studies. Given that cardiovascular health is a contributing factor to brain health, and cardiovascular disease is a 
significant risk factor for various forms of dementia, the benefits of polyphenols may also extend to brain functions.

Intriguingly, the mechanistic basis by which polyphenols may contribute to brain health and cognition extends further than those associated with cardiovascular health to include antiinflammatory and anti-oxidative capabilities (Vauzour et al., 2017; Flanagan et al., 2020). In addition, the developing role of the gut microbiota and its interplay with plant bioactives offers a novel route by which physiological effects can be exerted (Kumar Singh et al., 2019; Catalkaya et al., 2020). Continuing to reveal the underlying biochemical processes to which these compounds exert their effects is essential to utilizing their potential in brain health and disease, however, the importance of evaluating these compounds clinically cannot be underestimated. In this review, we will examine the current evidence on polyphenols and their effects on brain health, focusing specifically on citrus polyphenols since these are one of the main groups of flavonoids in the European diet.

\section{PLANT POLYPHENOLS}

Synthesized by plants to form either structural/functional tissue, or as a defense mechanism against pathogens and herbivores, polyphenol describes a compound with a chemical structure containing one or more phenolic rings (Frank et al., 2020). Further classifications can be made based upon phenolic ring numbers, and associated structures binding these rings together (e.g., oxygenated heterocycle) (Cutrim and Cortez, 2018). With an estimated 15,000 varieties currently identified, flavonoids represent a considerably diverse class of polyphenol (Xiao, 2017). The structural basis of the flavonoid is a 15-carbon atom structure, forming a C6-C3-C6 heterocyclic skeleton, consisting of two benzene rings, linked to a heterocyclic ring (flavon ring). Ubiquitous in plant-based diets, flavonoids are the main polyphenolic component of citrus fruits. Differing heterocyclic ring oxidization gives rise to further subclasses: flavanones, flavonols, flavones, and anthocyanins (present in blood oranges). Citrus fruits (Rutaceae) such as oranges, grapefruit, lemons and limes are a particularly rich source of flavanones and are therefore sometimes called citroflavonoids. Flavanones are also present in other food sources such as seasoning herbs (e.g., rosemary, oregano, peppermint), but in much lower concentrations (Rothwell et al., 2013).

Hesperidin (hesperetin-7-O-rutinoside) is a major citrus flavanone found in all citrus fruits (Jadeja and Devkar, 2014), reaching concentrations of $25.8-38.3 \mathrm{~g} / \mathrm{kg}$ in sweet oranges (Iglesias-Carres et al., 2019) and up to $520 \mathrm{mg} / \mathrm{L}$ in orange juice (De Rycker et al., 2020). Hesperidin is also the most common of the flavonoid monomers in European diets with an intake of approximately $27 \mathrm{mg} /$ daily (ZamoraRos et al., 2015), although regional variations are observed (Wang et al., 2015a). Oranges and orange juices also contain naringenin-7-O-rutinoside (narirutin) in addition to smaller quantities of hesperetin-7-O-rutinoside-3'-O-glucoside, $4^{\prime}-O$ methyl-naringenin-7-O-rutinoside (didymin), and eriodictyol-7O-rutinoside (eriocitrin) (Kay et al., 2017). Such compounds are believed to be responsible for many citrus related biological actions, although several other bioactives are also present in various citrus fruit and juice sources (e.g., anthocyanins, flavonols, carotenoids, pectins), and are therefore likely to also possess bioactive properties (Figure 1).

\section{MARKERS OF BRAIN HEALTH AND FUNCTION}

Markers of brain health and disease are key to understanding and treating neurological conditions. Development of sophisticated neuroimaging techniques [e.g., Positron Emission topography (PET) and functional Magnetic Resonance Imaging (fMRI)] has enabled subtle differences in brain activity, metabolism and structure to be monitored and distinguished between healthy and disease states (Agosta et al., 2013). Future incorporation of artificial intelligence techniques, e.g., deep learning, will likely enhance the prospect and feasibility of early detection of neurological conditions (Noor et al., 2020). However, the cost, expertise, and scalability of neuroimaging limits widespread use. Therefore, biological markers, detectable in the periphery (commonly cerebral spinal fluid or blood) remain equally important, and better suited for screening purposes. Below we discuss some specific markers of brain health in more detail.

\section{Neurotrophins}

Neurotrophins are blood-brain barrier permeable signaling molecules with a crucial role in synaptic plasticity, neuronal cell survival and neurogenesis/synaptogenesis (Gibon and Barker, 2017). The presence of neurotrophins in the peripheral circulation makes them an ideal candidate for monitoring brain health and function. Of the neurotrophins, brain-derived neurotrophic factor (BDNF) has been the most extensively studied in the context of brain health, with peripheral levels associating with cognitive performance and neurological diseases (Nascimento et al., 2014; Küster et al., 2017; Palasz et al., 2020). BDNF levels have been reported to increase linearly to the age of 65 , after which their levels markedly decrease (Neshatdoust et al., 2016). Interestingly, this predominantly corresponds to various MRI measures of brain health and cognition, although a high level of inter-study heterogeneity exists (McPhee et al., 2020).

\section{Blood-Brain Barrier}

Loss of blood-brain barrier (BBB) integrity/function is considered an early process of neurodegenerative disease. Thus, markers of BBB permeability and/or breakdown are being actively explored as measures of brain health and disease risk. Leakiness of BBB can be determined using MRI (dynamic contrast-enhanced MRI) or PET with various tracers such as gadolinium or 18F-fluoro-2-deoxyglucose (FDG) respectively (Sweeney et al., 2018). Additionally, MRI (T2*-weighted and susceptibility-weighted imaging MRI) can detect a chronic accumulation of small blood products, 'microbleeds', thought to be linked to $\mathrm{BBB}$ breakdown. Peripheral markers of $\mathrm{BBB}$ function have also been established. CSF soluble platelet-derived 


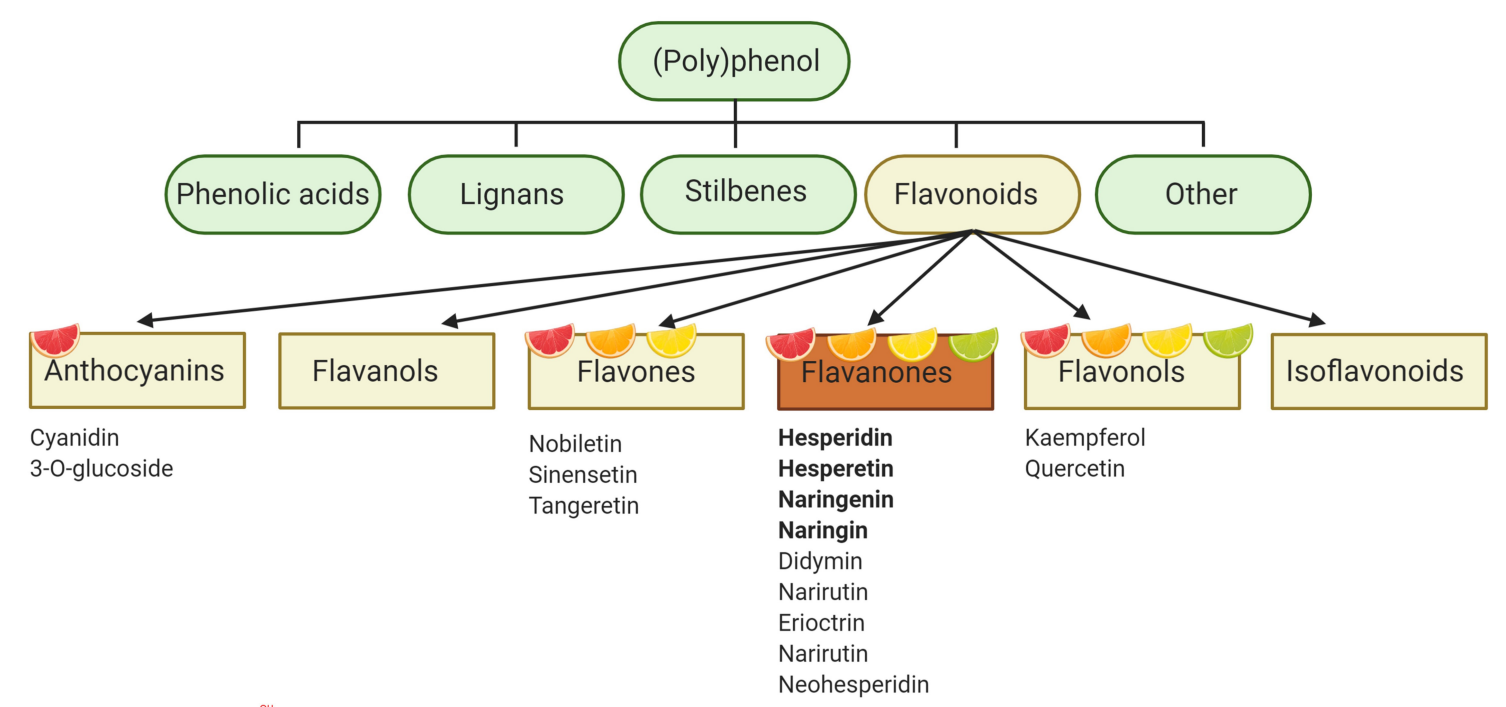

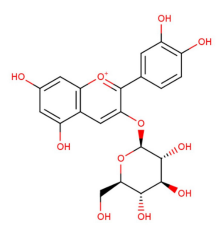

Cyanidin 3-0-glucoside

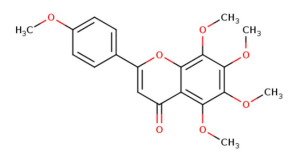

Tangeretin

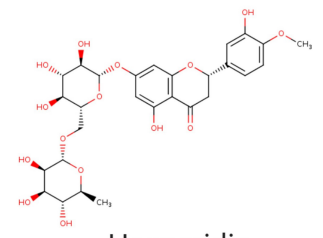

Hesperidin

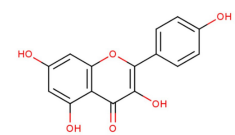

Kaempferol

FIGURE 1 | Polyphenol categorization - polyphenol describes a compound with a chemical structure containing one or more phenolic rings and can be classified into four main groups: phenolic acids, lignans, stilbenes, and flavonoids. Flavonoids represent the main polyphenolic component of citrus fruits and can be subdivided into six further subgroups. Flavanones are particularly abundant in citrus fruits and include hesperidin as one of the major flavanones. Figure created with BioRender.com.

growth factor receptor $\beta$ (sPDGFR $\beta)$, shed from pericytes (which unsheathe the endothelium of brain capillaries) in response to injury, correlates with neurodegenerative disease progression and BBB breakdown (Miners et al., 2019; Nation et al., 2019; Montagne et al., 2020). Similarly, the ratio of CSF albumin to serum albumin, is frequently used as an indicator of BBB breakdown (Musaeus et al., 2020), although conflicting results have been reported (Skillbäck et al., 2017). Less invasive methods including measuring circulating tight junction proteins (occludin, ZO-1 and CLDN5) have also been forwarded as BBB breakdown measures (Jiao et al., 2015; Zhu et al., 2017), whilst serum $14-\mathrm{kDa}$ monomeric form of transthyretin (TTR) and serum concentrations of the astrocytic protein S100B, have been suggested as measures of BBB damage (Marchi et al., 2004).

\section{Metabolic Function}

Metabolic homeostasis is particularly important for brain health and function due to the energy-demanding nature of the brain. Brain energy metabolism declines subtly during the aging process and prior to neurological disease diagnosis (Zilberter and Zilberter, 2017), and accumulating evidence demonstrates how this impairment of energy metabolism can exacerbate neurodegenerative disease progression (Cunnane et al., 2016; Camandola and Mattson, 2017). Neuroimaging techniques can effectively indicate metabolic disturbances. For example, FDG-PET is frequently utilized to determine brain glucose metabolism rates across the brain, which subsequently correlate to synaptic activity and disease risk (Shivamurthy et al., 2015). A multitude of other positron-emitting isotope tracers exist, including $\left[1-{ }^{11} \mathrm{C}\right]-\mathrm{DHA}$ (Yassine et al., 2017), and the incorporation of such tracers can hone in on specific aspects of brain metabolism which may be disease-specific. Furthermore, the ratio of $N$-acetylaspartate (NAA) and myoinositol (MI), two abundant metabolites in the human brain, has been reported to be a good predictor of mild cognitive impairment in cognitively normal older adults as measured by ${ }^{1} \mathrm{H}$ MRS in the posterior cingulate cortex (PCC) (Kantarci et al., 2013). Finally, peripheral measures of metabolic function such as insulin resistance and blood glucose, are routinely determined in clinical trials in which cognitive health is a primary output, and have been found to correlate with cognitive decline (Wium-Andersen et al., 2019), and brain atrophy (Erus et al., 2015).

\section{Inflammatory Markers}

Although neuroinflammation is an essential part of the brain response to infection or injury (Glass et al., 2010), sustained neuroinflammatory processes may contribute to the progressive neuronal damage observed in aging (Barrientos et al., 2015) and aged-related cognitive disorders (Heppner et al., 2015; Herrero et al., 2015). As resident macrophages, activated microglial cells have the capacity to synthesize a wide 
range of pro-inflammatory and anti-inflammatory cytokines and molecular mediators which contribute to the systemic inflammatory milieu and to the progression of neurodegenerative disease (Perry and Holmes, 2014). As an integral component of numerous neurological diseases, effective monitoring of neuroinflammation would be highly advantageous. PET may represent a viable option to achieve this (Chandra et al., 2019; Kreisl et al., 2020), along with a range of markers for activated microglia such as Translocator protein (TSPO) radioligands showing promise both in a preclinical setting and in humans. Optimization of TSPO tracers and identification of more specific neuroinflammatory tracers is, however, warranted to improve upon accuracy (Kreisl et al., 2020). Similar to peripheral markers of metabolic function, although not a direct marker of brain inflammation, circulating levels of the proinflammatory (IL- $1 \alpha$, IL- $1 \beta$, IL- 6, TNF- $\alpha$ ) and anti-inflammatory cytokines (IL-1ra and IL-10) indicate a chronic inflammatory environment and probably contribute to brain inflammation. Again, these measures frequently correlate with measures of brain health and disease such as cognitive performance (Magalhães et al., 2018; Contreras et al., 2020; Ribeiro-Santos et al., 2020).

\section{Cognitive Testing and Sensory Parameters}

As well as biological and neuroimaging techniques, brain health can be determined through cognitive assessment. For this, a wide range of tests can be employed, usually in combination as a battery, to address multiple aspects of cognition such as memory, language, executive functions and attention. Memory, a common target of numerous neurodegenerative disorders, can be separated into specific memory domains, enabling researchers to pinpoint disruptions to specific brain systems (e.g., episodic memory; hippocampaldiencephalic system, working memory; dorsolateral prefrontal cortex, semantic memory; anterior temporal lobe, executive functioning; frontal lobe and beyond) (Kipps and Hodges, 2005). Traditionally, such examination has required extensive clinical testing, performed by trained individuals (Kipps and Hodges, 2005), however, recent advancements in technology, e.g., smartphones and internet-based cognitive assessment tools may enable screening to take place outside of a clinical setting (Coutrot et al., 2019; Hays et al., 2019; Sternin et al., 2019).

Finally, it is worth briefly mentioning sensory parameters, particularly olfaction, which are potentially underrated, and certainly overlooked markers of brain health, despite consistent reports of their predictive capacity in cognitive decline (Brai et al., 2020).

\section{BIOAVAILABILITY OF CITRUS FLAVONOIDS}

The extent to which citrus flavanones may exert their biological action is strongly related to their bioavailability which can be affected by many factors including the structure of the compound, the food matrix, or host factors (age, sex, genetic polymorphism, gut microbiota etc.) (Kay et al., 2017; Morand and Tomás-Barberán, 2019). There is an apparent difference between the absorption of glycosides and aglycones with the glycosylation of flavanones increasing their hydrophilicity therefore abolishing passive diffusion and thus lowering their bioavailability (Najmanová et al., 2019). For example, following oral administration of hesperidin, plasma concentrations of flavanone conjugates (e.g., hesperetin-glucuronides and sulfoglucuronides) were detected after $3 \mathrm{~h}$ and reached a peak between $5 \mathrm{~h}$ and $7 \mathrm{~h}$ (Manach et al., 2003), highlighting that the main site for flavanone absorption is the small intestine and the colon. Such results were further confirmed by a more recent study where fasted participants, aged 51-69 years, received either orange juice or a hesperidin supplement (both providing $320 \mathrm{mg}$ hesperidin) versus a control (all matched for sugars and vitamin $\mathrm{C}$ content). Total plasma flavanone metabolite concentrations were significantly higher $5 \mathrm{~h}$ after the orange juice intervention than after control with hesperidin-glucuronide and naringenin-7-O-glucuronide, largely contributing to the total plasma flavanone concentration. Unexpectedly, no significant concentration of hesperidin metabolites was observed at $5 \mathrm{~h}$ following the hesperidin supplement intake, which may highlight the importance of food matrix in the bioavailability of these compounds (Schär et al., 2015). In addition to the main phase II metabolites found in the systemic circulation, the intestinal microflora further degrades aglycones into smaller phenolics. For example, the main degradation product of hesperetin is 3-(3'-hydroxy-4'-methoxyphenyl)propionic acid, although many other compounds have also been reported (see Kay et al., 2017; Najmanová et al., 2019 for extensive reviews of flavanones metabolism).

Flavanone bioavailability can differ across citrus source and/or form. For example, the bioavailability of hesperidin, indirectly determined from excreted hesperetin (main hesperidin metabolite marker) is comparable for both whole orange fruit $(1.5 \%)$ and orange juice $(2.9 \%)$, despite the considerably higher flavanone content of the fruit (Aschoff et al., 2016), reflecting potential absorption and metabolic differences. Fresh and packaged orange juice, on the other hand, appear to have relatively similar metabolic kinetics in regards to flavanones, and therefore the higher flavanone content found in packaged juice (machine pressed) translates to a threefold greater flavanone status (Silveira et al., 2014).

In addition, a large inter-individual variability in the bioavailability of citrus flavanones has been reported with high, medium, and low flavanone metabolite excreters identified following intake of citrus flavanones and citrus juices (Nielsen et al., 2006; Brett et al., 2008; Vallejo et al., 2010; Aschoff et al., 2016). One of the key factors in this variability may reside in the host-microbiota diversity necessary for the conversion of the flavanone rutinoside into their aglycone forms (Stevens et al., 2019), although the impact of lifestyle factors such as exercise have also been reported (Pereira-Caro et al., 2017). Despite the demonstrated high inter-individual variability in citrus flavanones metabolism, limited evidence is currently 
available regarding the role of the gut microbiota composition and no correlation with the effects on brain health biomarkers has been demonstrated.

When considering the bioavailability of citrus flavonoids from a neurological perspective, one must also consider the compounds ability to traverse the $\mathrm{BBB}$. The extent to which specific citrus polyphenols cross the $\mathrm{BBB}$ remains to be fully categorized, however, evidence to date in the form of in vitro cellular models (e.g., RBE4, b.END5, and hCMEC/D3) (Youdim et al., 2003; Yang W. et al., 2014), and preclinical approaches (Peng et al., 1998; Tsai and Chen, 2000; Youdim et al., 2004) indicate that citrus flavonoids, namely; hesperetin, naringenin, as well as their relevant metabolites, are able to reach the brain. Whether these models fully translate to humans, particularly when ingested with other complex food sources is yet to be proven, however, pursuing this question will undoubtedly enhance our understanding of citrus polyphenols in the context of brain health and disease, elucidating the extent to which their bioactivity stems from direct interaction with the brain parenchyma.

\section{MICROBIOTA: GUT: BRAIN AXIS}

A healthy gastrointestinal tract is a nutrient-rich environment hosting approximately 100 trillion microbes (Lin and Zhang, 2017), that constitute the gut microbiota. Gut microbial composition is increasingly recognized as a central factor in health and disease, protecting the intestinal gut barrier and preventing the establishment of pathogenic microorganisms. The gut microbiota also provides a large repertoire of genes, antigens and metabolites that can regulate immune and metabolic functions. The gut-brain axis describes a bidirectional system that encompasses both neuro-immune (Teratani et al., 2020) and neuro-endocrine communication as well as a direct neuronal connection (vagus nerve) (Tan et al., 2020), with each mode of transmission receiving microbial modulation (Lv et al., 2019; Wang et al., 2019). The gut-brain axis has rapidly emerged in recent decades, with an influx of literature implicating gut health and microbial dysbiosis with specific neurological diseases/conditions, although the multifactorial nature and complexity of these diseases/conditions has left mechanistic validation somewhat lagging (Sherwin et al., 2018). Indeed, further elucidation of the mechanistic as well as the determination of overall therapeutic validity are still required (Peterson, 2020). An area in which mechanistic evidence is beginning to gain traction is the synthesis and/or regulation of metabolites by the gut-microbiota, with many derivative metabolites established as neurochemicals or possessing neuromodulatory properties. Short-chain fatty acids (SCFA) are a relatively well-characterized example of this (Dalile et al., 2019) and are produced via fermentation of complex carbohydrates (dietary fibers). SCFA levels are diminished in response to antibiotic treatment (Høverstad et al., 1986) and similarly low levels tend to associate with conditions such as depression, Alzheimer's (AD) and Parkinson's disease (Dalile et al., 2019). For example, the SCFA propionate was recently reported to inhibit pathways associated with nonspecific microbial infections via a CD14-dependent mechanism, to suppress the expression of LRP-1 and to protect the BBB from oxidative stress via NRF2 (NFE2L2) signaling (Hoyles et al., 2018). The influence of metabolites such as SCFA produced by our 'gut-biofactory' has been extensively reviewed by SkoniecznaŻydecka et al. (2020).

The considerable inter-individual variability of the gut microbiota combined with its apparent modifiable/dynamic nature has led to it being identified as a therapeutic target. The fact that beneficial shifts could be achieved through noninvasive, relatively safe interventions, e.g., prebiotics, make it an attractive target. The gut microbiota is both modulated by, and modulates, polyphenolic compounds (Koudoufio et al., 2020). Citrus polyphenols appear to be no exception, with neohesperidin recently shown to reverse high-fat-diet-induced intestinal microbiota dysbiosis by increasing general microbial diversity as well as specific strains including Bacteroidetes and Firmicutes (Lu et al., 2020). A similar experiment in which hesperidin was administered exerted similar prebiotic effects with treated mice displaying improved metabolic maker profile (Guirro et al., 2020). These beneficial hesperidin mediated effects are in agreement with an earlier experiment, which interestingly reported upon the concomitant immunomodulatory actions (Estruel-Amades et al., 2019). In healthy volunteers, continuous consumption of commercial pasteurized orange juice for 2 months improved blood biochemical parameters, such as lowdensity lipoprotein-cholesterol, glucose, and insulin sensitivity and positively modulated the composition and metabolic activity of the microbiota, increasing the population of fecal Bifidobacterium spp. and Lactobacillus spp. (Lima et al., 2019). In addition, daily consumption of $500 \mathrm{~mL}$ of two Brazilian orange juices (e.g., Cara Cara and Bahia juices) for 7 days increased the abundance of Mogibacteriaceae, Tissierellaceae, Veillonellaceae, Odoribacteraceae, and Ruminococcaceae families in healthy volunteers (Brasili et al., 2019). Furthermore, daily consumption of $300 \mathrm{ml}$ of orange juice for 60 days affected the levels of Lactobacillus spp., Akkermansia spp., and Ruminococcus spp. and improved the glycemia and lipid profiles in 20-35 years old healthy female volunteers (Fidélix et al., 2020). These recent reports emphasize the prebiotic potential of citrus polyphenols, particularly in metabolic disease, with no specific information related to brain functions (e.g., gut-brain axis). With this being said, it must be mentioned that current evidence has been predominantly provided through preclinical and in particular, rodent experiments. Therefore, current perspectives derived from these studies should be cautiously interpreted until validated through robust clinical trials since fundamental human to mouse differences, such as microbial composition and gut physiology, may render direct translation inappropriate.

\section{METHOD}

A non-systematic search was performed in PubMed using the following keywords to retrieve preclinical and clinical data: [(Dementia OR depression OR Alzheimer OR Parkinson 
OR neurodegeneration OR neuroinflammation) AND (citrus polyphenol OR flavanone OR hesper* OR naring*) AND (cognition OR brain)]. Articles in English or with English abstracts were retrieved. All evidence was read and chosen based on the Authors' evaluation of relevance. The search was concluded in August 2020 and updated in November 2020, and included all articles present in PubMed, without temporal limits.

\section{PRECLINICAL EVIDENCE FOR CITRUS FLAVONOIDS AND BRAIN HEALTH/FUNCTION}

Neurodegeneration describes a process of progressive cell dysfunction and eventual neuronal cell death. In diseases such as late-onset Alzheimer's Disease (LOAD) this process can span decades. Although usually categorized based upon distinct pathologies, the overall process of neurodegeneration is one that is multi-factorial, with many neurodegenerative diseases sharing common pathogenic mechanisms underpinning disease progression (Jellinger, 2010), potentially explaining the lack of efficacy of single target drugs. These common mechanisms associated with neurodegeneration include: neuroinflammatory/neuroimmune responses, oxidative stress, impaired bioenergetics/mitochondrial dysfunctions, dysfunction of neurotrophins and abnormal protein dynamics. The mechanistic understanding of citrus polyphenols in brain health stems largely from preclinical and in vitro studies. The focus of these preclinical studies often centers on mitigating specific disease pathologies. Indeed, a wealth of preclinical evidence has highlighted the multifaceted nature of citrus polyphenols neurologically. Interestingly, as highlighted in Table 1, the major citrus polyphenols share common mechanistic actions, overlapping considerably with the aforementioned deficits associated with neurodegeneration. Below we discuss these mechanisms in more details focusing on the key citrus flavonoids and in particular on the flavanones hesperidin and naringin along with their aglycone forms (i.e., hesperetin and naringenin) and the flavonol, kaempferol.

\section{Anti-oxidative}

Oxidative stress is a well-established contributing factor in neurological disorders (Michalska and León, 2020) with high metabolic activity combined with a lack of antioxidant defense capability leaving the brain particularly susceptible. Although citrus polyphenols reportedly demonstrate free radical scavenger capacity in vitro (Di Meo et al., 2013), the current literature search unanimously highlighted their ability to stimulate the endogenous antioxidant defense machinery, with superoxide dismutase (SOD), catalase (CAT), glutathione (GSH), glutathione S-transferases (GST), glutathione reductase (GR) and glutathione peroxidase (GPx) activation common across all the citrus polyphenols reviewed (Table 1). Effective dosage ranged from as little as $5 \mathrm{mg} / \mathrm{kg}$ up to $300 \mathrm{mg} / \mathrm{kg}$, however, dose dependant effects were established in a number of studies, with greater doses usually eliciting greatest effects
(Table 1). Activation coincided with subsequent reduction of reactive oxygen species (ROS) such as hydrogen peroxide $\left(\mathrm{H}_{2} \mathrm{O}_{2}\right)$, nitric oxide $(\mathrm{NO})$ and other oxidative markers namely malondialdehyde (MDA) and thiobarbituric acid reactive substances (TBARS). Upregulation of the transcription factor NRF2 features in a number of these studies (Bai et al., 2014; Sugumar et al., 2019; Welbat et al., 2020), and is likely central to this polyphenol mediated anti-oxidative system through the activation of the antioxidant response element (Vauzour, 2012). As alluded to, activation of the antioxidant defense machinery was generally consistent across the flavonoids with only a few discrepancies found, mainly confined to naringenin and hesperetin, where occasionally no effect was detected (Chtourou et al., 2014; Kheradmand et al., 2018; Mani et al., 2018). A few studies reported the opposite effect for naringenin with SOD and CAT reduced (Chtourou et al., 2014; Haider et al., 2020). This reduction associated with naringenin, which is believed to be able to cross the BBB (Youdim et al., 2003), may relate back to its free radical scavenging capabilities, or arise as a result of the differing disease models used. In addition to the more common antioxidant machinery influenced, hesperidin and hesperetin upregulated Haem-oxygenase (HO-1) and downregulated the superoxide radical generating enzyme Xanthine Oxidase $(\mathrm{XO})$ respectively (Ashafaq et al., 2014).

\section{Anti-inflammatory}

The immunomodulatory capabilities of citrus polyphenols within the brain are similarly evident, and are in some cases coupled to anti-oxidative mechanisms (e.g., HMGB1/RAGE, Yu et al., 2015). Again, the molecular targets with which the reviewed citrus polyphenols interact appear to be consistent, with reduction of pro-inflammatory cytokines IL- $1 \beta$, IL-2, IL-6, IFN- $\gamma$, and TNF- $\alpha$, particularly prolific in the literature (Table 1). This is likely mediated through the mitigation of hyperactive immune cells as is suggested by the reduction of GFAP, and NF- $\kappa B$ which governs chemokine and inflammatory mediator transcription (Fu et al., 2019; Zhou et al., 2020). In contrast to evidence on anti-oxidative effects, there was little indication to suggest lack of effect across any of the reviewed flavonoids, supporting the notion that neuro-inflammatory modulation is inherent across all citrus flavonoids. As with anti-oxidative effects, effective dosage had a considerable range of $20-300 \mathrm{mg} / \mathrm{kg}$, with efficacy remaining even at lower doses $25-50 \mathrm{mg} / \mathrm{kg}$. This latter dose equates to $145-290 \mathrm{mg}$ human equivalent dose in a $70 \mathrm{Kg}$ adult, if the animal is a mouse (Nair and Jacob, 2016), which is achievable through consumption of $0.5-0.75 \mathrm{~L}$ of orange juice (De Rycker et al., 2020).

\section{Bioenergetic}

Citrus flavonoids appear to ameliorate mitochondrial dysfunction - damage to the mitochondria which may be due to exogenous factors and which can predispose individuals to certain neurodegenerative conditions. As with anti-inflammatory properties, the impact was consistent across the reviewed studies with reported dosages of $25-200 \mathrm{mg} / \mathrm{kg}$ resulting in an increase of mitochondrial respiratory chain complexes 
TABLE 1 | Overview of the molecular mechanisms underlying the impact of citrus flavonoids on brain health and disease in preclinical models.

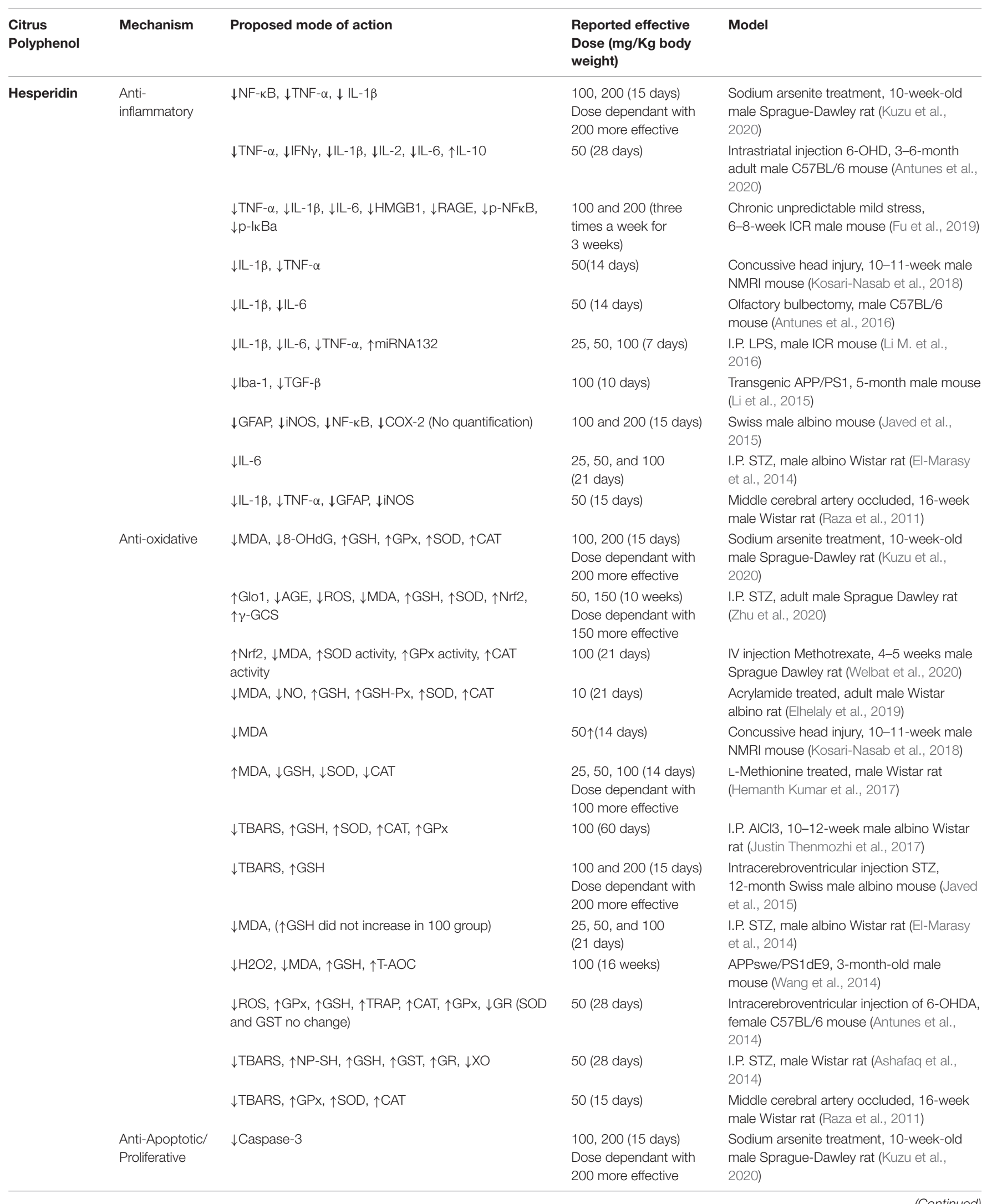


TABLE 1 | Continued

\section{Citrus Mechanism Proposed mode of action \\ Polyphenol}

BDNF, $\downarrow p 21$ positive cells, $\uparrow \mathrm{DCX}$

$\uparrow B D N F, \uparrow N G F, \uparrow N T 3$

个Ki-67, $\uparrow B r d U, ~ \uparrow D C X$

$\uparrow B D N F, \uparrow p-T r k B$

$\uparrow B D N F$

$\uparrow B D N F, \uparrow N G F$

$\downarrow B A X, \uparrow B C L-2$

$\uparrow p-E R K, \uparrow B D N F(E R K$ dependent)

个BDNF

Bioenergetic $\quad \downarrow \mathrm{AChE}$

$\downarrow$ AChE

$\downarrow$ AChE activity

$\uparrow$ MT complex (I-IV) activity, $\uparrow$ MTT activity, $\uparrow$ GSK3b phos

$\downarrow$ AChE, $\uparrow \mathrm{Na}+-\mathrm{K}+$ ATPase

Proteinopathy

$\downarrow A \beta$ deposition

No change $A \beta$ deposition

BBB

dysfunction

Anti-

inflammatory

$\downarrow$ EB leakage, $\downarrow$ claudin-5 and ZO-1 disturbance

$\downarrow$ TLR4, $\downarrow$ GFAP, $\downarrow$ lba- $1, \downarrow$ TNF- $\alpha, \downarrow \| L-1 \beta, \downarrow p-N F-\kappa B$

$\downarrow$ TNF-a, $\downarrow \|-1 b, \downarrow$ iNOS

$\downarrow$ GFAP, $\downarrow N F-\kappa B$

Anti-oxidative

$\downarrow M D A, \uparrow G S H, \uparrow S O D, \uparrow C A T$ (50 only), $\downarrow N O$ (50 only)

$\uparrow N r f-2, \uparrow H O-1 \downarrow R O S, \downarrow L P O$

$\downarrow$ MDA, (GSH, CAT, SOD, GPx, GRx No change) (however effect significant in Nano form)

$\downarrow$ TBARS, $\downarrow$ LOOH and $\downarrow$ Protein carbonyl content (LPO), $\quad 40$ (21 days)

$\downarrow \mathrm{OH}-, \downarrow N O, \uparrow \mathrm{GSH}, \uparrow \mathrm{TSH}, \uparrow \mathrm{SOD}, \uparrow \mathrm{CAT}, \uparrow \mathrm{GPX}$ and $\uparrow$ GST

$\downarrow \mathrm{MDA}, \uparrow \mathrm{CAT}, \uparrow \mathrm{GSH}$, (NO No change)

Proliferative

100 (21 days)
个BDNF

$\downarrow p-J N K, \downarrow$ Bax:Bcl2, $\downarrow$ Caspase-3
50 (1 week)

5, 50 (3 days)

Reported effective

Dose (mg/Kg body

weight)

100 (21 days)

50 (28 days)

$100,200(3 x$ in

21 days)

100 and 200 (21 days)

50 (14 days)

100 (60 days)

25, 50 (3 weeks)

25, 50, and 100

(21 days)

100, 200 (15 days)

Dose dependant with

200 more effective

50 (14 days)

100 and 200 (15 days)

100 (16 weeks)

50 (28 days)

100 (10 days)

100 (16 weeks)

10 (1 dose prior to

$\mathrm{MCAO})$

50 (5 weeks)

20 (8 days)

50 (1 week)

5, 50 (3 days)

50 (5 weeks)

10, 20 (3 weeks)

50 (5 weeks)
Model

I.V injection Methotrexate, male Sprague Dawley rat (Welbat et al., 2020)

Intrastriatal injection 6-OHD, 3-6-month adult male C57BL/6 mouse (Antunes et al., 2020)

IV injection Methotrexate, male Sprague Dawley rat (Naewla et al., 2019)

Chronic unpredictable mild stress, 6-8-week male ICR mouse (Fu et al., 2019)

Concussive head injury, 10-11-week male NMRI mouse (Kosari-Nasab et al., 2018)

Olfactory bulbectomy, male C57BL/6 mouse (Antunes et al., 2016)

IP AICl3, 10-12-week male albino Wistar rat (Justin Thenmozhi et al., 2017)

Chronic mild stress, 5-week male ICR mouse (Li C. F. et al., 2016)

I.P. STZ, male albino Wistar rat (El-Marasy et al., 2014)

Sodium arsenite treatment, 10-week-old male Sprague-Dawley rat (Kuzu et al., 2020)

Olfactory bulbectomy, male C57BL/6

mouse (Antunes et al., 2016)

Intracerebroventricular injection STZ 12-month Swiss male albino mouse (Javed et al., 2015)

APPswe/PS1dE9, 3-month-old male mouse (Wang et al., 2014)

I.P. STZ, male Wistar rat (Ashafaq et al., 2014)

Transgenic APP/PS1-21, 5-month male mouse (Li et al., 2015)

Three-month-old male, APPswe/PS1dE9 mouse (Wang et al., 2014)

Left middle cerebral artery occlusion, male 8 weeks ICR mouse (Lee et al., 2020)

I.P. LPS, 7/8 weeks male C57BL/6N mouse (Muhammad et al., 2019)

Hippocampal injection Kainic Acid, mouse (Kwon et al., 2018)

Intrastriatal injection 6-OHDA, male adult Wistar rat (Kiasalari et al., 2016)

I.P. scopolamine, male albino mouse (Ishola et al., 2019)

I.P. LPS, 7-8-week male C57BL/6N mouse (Muhammad et al., 2019)

I.P. injection STZ, male Wistar rat (Kheradmand et al., 2018)

Subcutaneous injection of Cadmium, male albino Wistar rat (Shagirtha et al., 2017)

Intrastriatal injection of 6-OHDA, male adult Wistar rat (Kiasalari et al., 2016)

I.P. scopolamine, male albino mouse (Ishola et al., 2019)

I.P. LPS 7-8-week, male C57BL/6N mouse (Muhammad et al., 2019) 
TABLE 1 | Continued

\begin{tabular}{|c|c|c|c|c|}
\hline $\begin{array}{l}\text { Citrus } \\
\text { Polyphenol }\end{array}$ & Mechanism & Proposed mode of action & $\begin{array}{l}\text { Reported effective } \\
\text { Dose (mg/Kg body } \\
\text { weight) }\end{array}$ & Model \\
\hline & & $\downarrow$ Bax, $\downarrow$ cytochrome c, $\downarrow$ caspase 3 and $9, \uparrow \mathrm{Bcl} 2$ & 40 (21 days) & $\begin{array}{l}\text { Subcutaneous injection of Cadmium, male } \\
\text { albino Wistar rat (Shagirtha et al., 2017) }\end{array}$ \\
\hline & & $\uparrow B c 12, \downarrow D N A$ fragmentation & 50 (1 week) & $\begin{array}{l}\text { Intrastriatal injection of 6-OHDA, male adult } \\
\text { Wistar rat (Kiasalari et al., 2016) }\end{array}$ \\
\hline & & $\uparrow A C h E, \uparrow A T P a s e s, \uparrow M T$ complex (I-IV) activity & 40 (21 days) & $\begin{array}{l}\text { Subcutaneous injection of Cadmium, male } \\
\text { albino Wistar rat (Shagirtha et al., 2017) }\end{array}$ \\
\hline & Proteinopathy & $\uparrow$ Cathepsin, trypsin and pronase activity & 40 (21 days) & $\begin{array}{l}\text { Subcutaneous injection of Cadmium, male } \\
\text { albino Wistar rat (Shagirtha et al., 2017) }\end{array}$ \\
\hline \multirow[t]{17}{*}{ Naringenin } & $\begin{array}{l}\text { Anti- } \\
\text { inflammatory }\end{array}$ & $\downarrow$ GFAP, $\downarrow$ TNF $-\alpha, \downarrow I L-1 \beta, \uparrow I L 10$ & 250-300 (10 weeks) & $\begin{array}{l}\text { High-fat diet-fed, 10-month SAMP8 male } \\
\text { mouse (Zhou et al., 2020) }\end{array}$ \\
\hline & & $\downarrow N F \kappa B, \downarrow T N F \alpha, \downarrow C O X 2, \downarrow$ NOS $, \downarrow T L R 4, \downarrow$ GFAP & 100 (7 days) & $\begin{array}{l}\text { I.P. LPS, male albino Wistar rat } \\
\text { (Khajevand-Khazaei et al., 2018) }\end{array}$ \\
\hline & & $\downarrow \mathrm{IL}-1 \beta, \downarrow \mathrm{TNF}-\alpha($ not $25 \mathrm{mg} / \mathrm{kg})$ & $25,50,100$ (5 days) & $\begin{array}{l}\text { I.P. MPTP, male C57BL/6J mouse (Mani } \\
\text { et al., 2018) }\end{array}$ \\
\hline & & $\downarrow N F \kappa B, \downarrow$ NOS $, \downarrow C O X 2, \downarrow T N F-\alpha, \downarrow \| L-1 \beta$ & 50 (21 days) & $\begin{array}{l}\text { Middle cerebral artery occlusion, 16-week } \\
\text { male Wistar rat (Raza et al., 2013) }\end{array}$ \\
\hline & Anti-oxidative & $\uparrow S O D, \uparrow G S H, \downarrow M D A, \downarrow N O$ & 250-300 (10 weeks) & $\begin{array}{l}\text { High-fat diet-fed, } 10 \text { months SAMP8 male } \\
\text { mouse (Zhou et al., 2020) }\end{array}$ \\
\hline & & $\downarrow$ SOD activity, $\uparrow \mathrm{CAT}, \uparrow \mathrm{GPx}, \uparrow \mathrm{GSH}, \downarrow \mathrm{MDA}$ & 50 (14 days) & $\begin{array}{l}\text { I.P. } \mathrm{AlCl}_{3}+\text { D-gal injection, young albino } \\
\text { Wistar rat (Haider et al., 2020) }\end{array}$ \\
\hline & & $\uparrow \mathrm{GSH}, \uparrow \mathrm{GST}, \downarrow \mathrm{MDA}, \downarrow$ Protein carbonyl content & 100 (15 days) & $\begin{array}{l}\text { Oral MeHg, 5-week male Swiss albino } \\
\text { mouse (Krishna Chandran et al., 2019) }\end{array}$ \\
\hline & & $\uparrow \mathrm{CAT}, \uparrow \mathrm{SOD}, \uparrow \mathrm{GSH}, \downarrow \mathrm{MDA}, \uparrow \mathrm{GPx}, \downarrow \mathrm{MDA}$ & 50 (16 days) & $\begin{array}{l}\text { Young adult male Albino Wistar rat (Liaquat } \\
\text { et al., 2018) }\end{array}$ \\
\hline & & $\downarrow \mathrm{MDA}$, (SOD and NO No change) & 100 (single dose) & $\begin{array}{l}\text { Dorsal hippocampal A } \beta \text { injection, adult male } \\
\text { Wistar rat (Ghofrani et al., 2015) }\end{array}$ \\
\hline & & $\downarrow N O D 2, \downarrow R I P 2, \downarrow M M P-9$ & 100 (4 days) & $\begin{array}{l}\text { Permanent middle cerebral artery } \\
\text { occlusion, male Sprague-Dawley rats (Bai } \\
\text { et al., 2014) }\end{array}$ \\
\hline & & $\begin{array}{l}\downarrow R O S, \downarrow N O, \downarrow M D A, \downarrow P C O, \uparrow \text { ascorbic acid, } \uparrow \text { total thiol } \\
\text { groups } \downarrow \text { SOD, } \downarrow \text { CAT, (GPx No change) }\end{array}$ & 50 (4 weeks) & $\begin{array}{l}\text { I.P. iron }(\mathrm{Fe}) \text { injection, 10-week male Wistar } \\
\text { rat (Chtourou et al., 2014) }\end{array}$ \\
\hline & & $\uparrow N r f 2$ and downstream ARE genes, $\downarrow R O S, \uparrow G S H$ & 70 (4 days) & $\begin{array}{l}\text { Intastriatal 6-OHDA, 10-week male } \\
\text { C57BL/6 mouse (Lou et al., 2014) }\end{array}$ \\
\hline & & $\downarrow$ TBARS $, \uparrow \mathrm{GSH}, \downarrow \mathrm{NO}, \downarrow \mathrm{MPO}$ activity, $\uparrow \mathrm{SOD}$ activity & 50 (21 days) & $\begin{array}{l}\text { Middle cerebral artery occlusion, 16-week } \\
\text { male Wistar rat (Raza et al., 2013) }\end{array}$ \\
\hline & $\begin{array}{l}\text { Anti-Apoptotic/ } \\
\text { Proliferative }\end{array}$ & $\uparrow B D N F, \uparrow S h h$ & 50 (5 weeks) & $\begin{array}{l}\text { Chronic unpredictable mild stress, adult } \\
\text { male Wistar rat (Tayyab et al., 2019) }\end{array}$ \\
\hline & & $\uparrow B D N F$ & 50, 100 (14 days) & $\begin{array}{l}\text { Bilateral olfactory bulbectomy, 3-month } \\
\text { BALB/c male mouse (Bansal et al., 2018) }\end{array}$ \\
\hline & & $\downarrow D N A$ Fragmentation & 100 (single dose) & $\begin{array}{l}\text { Dorsal hippocampal A } \beta \text { injection, adult male } \\
\text { Wistar rat (Ghofrani et al., 2015) }\end{array}$ \\
\hline & & $\downarrow p-J N K, \downarrow p-p 38$ & 70 (4 days) & $\begin{array}{l}\text { Intastriatal 6-OHDA, 10-week male } \\
\text { C57BL/6 mouse (Lou et al., 2014) }\end{array}$ \\
\hline
\end{tabular}


TABLE 1 | Continued

\begin{tabular}{lll}
\hline Citrus & Mechanism Proposed mode of action \\
Polyphenol &
\end{tabular}

个BDNF (Hippocampus)

Bioenergetic

$\uparrow$ Ach, $\downarrow$ AChE, $\downarrow$ AChE activity

$\uparrow$ MT complex (I-IV) activity, $\downarrow$ MT DNA damage

$\downarrow K Y N: T R P$ ratio

$\downarrow$ AChE activity

$\uparrow$ Ach, $\downarrow$ AChE

$\uparrow \mathrm{Na}+-\mathrm{K}+$ ATPase, $\uparrow$ AChE activity

$\uparrow P P A R \gamma \uparrow I D E, \uparrow I N S, \uparrow I N S R$

Proteinopathy

Anti-

inflammatory

Anti-oxidative

Anti-Apoptotic/

Proliferative

Bioenergetic $\downarrow$ Soluble and insoluble A $\beta$ 40, A $\beta$ 42, $\downarrow$ APP, $\downarrow$ BACE1, $\downarrow P$-tau, $\downarrow$ P-GSK

$\downarrow \alpha S Y N(100 \mathrm{mg} / \mathrm{kg}$ only), $\downarrow \alpha S Y N+$ cells

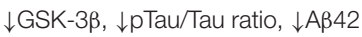

$\downarrow$ GFAP

$\downarrow$ TNF- $\alpha, \downarrow I L-1 \beta, \downarrow I L-6, \downarrow N F-k B$

$\downarrow \mathrm{TNF}-\alpha$

$\downarrow N F-k B, \downarrow G F A P$

$\downarrow$ iNOS, $\downarrow T N F-\alpha, \downarrow N F-\kappa B$

$\downarrow \mathrm{TNF}-\alpha, \downarrow \mathrm{IL}-1 \beta$

$\uparrow S O D, \uparrow G S H, \downarrow M D A, \downarrow N O$

$\downarrow$ MDA (reduced by $10 \mathrm{mg} / \mathrm{kg}$ also), $\uparrow \mathrm{CAT}, \uparrow \mathrm{GSH}$, $\uparrow S O D$ (40 mg/kg only), $\downarrow N O$

$\downarrow \mathrm{MDA}, \downarrow \mathrm{PCO}, \downarrow \mathrm{H} 2 \mathrm{O} 2, \downarrow R O S, \downarrow N O$ (50 and 100 only), $\uparrow$ SOD, $\uparrow$ CAT, $\uparrow$ GSH, $\uparrow$ GPX

$\downarrow$ ROS

$\downarrow \mathrm{MDA}, \downarrow N \mathrm{NO}, \uparrow \mathrm{GSH}, \uparrow \mathrm{SOD}, \uparrow \mathrm{CAT}$

个DCX

$\downarrow$ Bax, $\uparrow$ Bcl-2, $\downarrow$ Caspase-3, $\uparrow$ PPAR- $\gamma$

$\uparrow \mathrm{MT}$ complex (I-IV)

$\downarrow$ AChE activity, $\uparrow \mathrm{Na}+-\mathrm{K}+$ ATPase, $\uparrow$ Ca2 + ATPase, $\uparrow \mathrm{Mg} 2+$ ATPase (increased by $25 \mathrm{mg} / \mathrm{kg}$ also)

$\uparrow$ MT membrane potential, $\uparrow$ ATP levels, $\uparrow$ AMPK

\section{Reported effective \\ Dose (mg/Kg body \\ weight)}

10, 20 (3 Weeks)

50 (14 days

100 (15 days)

50, 100 (14 days)

100 (7 days)

50 (16 days)

50 (4 weeks)

25, 50, 100 (21 days)

250-300 (10 weeks)

25, 50, 100 (5 days)

25, 50, 100 (21 days)

100 (14 days)

40 and $80 \uparrow$ (28 days)

20 and 40 (28 days)

80 (14 days)

25, 50100 (5 weeks)

50, 100, and 200

(21 days)

40 and 80 (28 days)

20 and 40 (28 days)

25, 50, and 100

(5 weeks)

100 (20 weeks)

50, 100 , and 200

(21 days)

100 (14 days)

40 and 80 (28 days)

40 and 80 (28 days)

50 and 100 (5 weeks)

100 (20 weeks)

\section{Model}

Chronic unpredictable mild stress, 5-wee male ICR mouse (Yi et al., 2014)

I.P. $\mathrm{AlCl}_{3}+\mathrm{D}$-gal injection, young albino Wistar rat (Haider et al., 2020)

Oral MeHg, 5-week male Swiss albino mouse (Krishna Chandran et al., 2019)

Bilateral olfactory bulbectomy, 3-month BALB/c male mouse (Bansal et al., 2018)

I.P. LPS, male albino Wistar rat (Khajevand-Khazaei et al., 2018)

Young adult male albino Wistar rat (Liaquat et al., 2018)

I.P. injection iron (Fe), 10 week male Wistar rat (Chtourou et al., 2014)

Intracerebroventricular injection STZ, adult male Sprague Dawley rat (Yang W. et al., 2014)

High-fat diet-fed, 10-month SAMP8 male mouse (Zhou et al., 2020)

I.P. injection MPTP, male C57BL/6J mouse (Mani et al., 2018)

Intracerebroventricular injection STZ, adult male Sprague Dawley rat (Yang W. et al., 2014)

STZ treated, C57BL/6, 9-week male mouse (Okuyama et al., 2018)

Intrastriatal injection of quinolinic acid, adult male Sprague-Dawley rat (Cui et al., 2018)

Intastriatal injection collagenase, adult female Wistar rat (Singh et al., 2017)

I.P. injection 3-nitropropionic acid, male Wistar rat (Gopinath and Sudhandiran, 2016)

Cisplatin exposed, middle aged Wistar rat (Chtourou et al., 2015)

Intracerebroventricular STZ, adult male Wistar rat (Sachdeva et al., 2014)

Intrastriatal injection quinolinic acid, adult male Sprague-Dawley rat (Cui et al., 2018) Intastriatal injection collagenase, adult female Wistar rat (Singh et al., 2017)

Cisplatin exposed, middle aged Wistar rat (Chtourou et al., 2015)

High-fat diet induced cognitive decline, male C57BL/6 mouse (Wang et al., 2015b)

Intracerebroventricular STZ, adult male Wistar rat (Sachdeva et al., 2014)

STZ treated, C57BL/6 male (9-week) mouse (Okuyama et al., 2018)

Intrastriatal injection of quinolinic acid, adult male Sprague-Dawley rat (Cui et al., 2018)

Intrastriatal injection of quinolinic acid, adult male Sprague-Dawley rat (Cui et al., 2018) Cisplatin exposed, middle aged Wistar rat (Chtourou et al., 2015)

High-fat diet induced cognitive decline, male C57BL/6 mouse (Wang et al., 2015b) 
TABLE 1 | Continued

\begin{tabular}{|c|c|c|c|c|}
\hline $\begin{array}{l}\text { Citrus } \\
\text { Polyphenol }\end{array}$ & Mechanism & Proposed mode of action & $\begin{array}{l}\text { Reported effective } \\
\text { Dose (mg/Kg body } \\
\text { weight) }\end{array}$ & Model \\
\hline & & $\downarrow$ AChE activity, $\uparrow$ MT complex (I-IV) activity & $\begin{array}{l}50,100, \text { and } 200 \\
\text { (21 days) }\end{array}$ & $\begin{array}{l}\text { Intracerebroventricular STZ, adult male } \\
\text { Wistar rat (Sachdeva et al., 2014) }\end{array}$ \\
\hline & Proteinopathy & $\downarrow$ Thr231 and Ser396 Tau phos & 100 (14 days) & $\begin{array}{l}\text { STZ treated, C57BL/6 male (9-week) } \\
\text { mouse (Okuyama et al., 2018) }\end{array}$ \\
\hline \multirow[t]{11}{*}{ Kaempferol } & $\begin{array}{l}\text { Anti- } \\
\text { inflammatory }\end{array}$ & $\begin{array}{l}\downarrow \text { lba- } 1, \downarrow T N F \alpha, \downarrow I L-1 \beta, \downarrow I L-5, \downarrow \| L-6, \downarrow \text { iNOS, } \downarrow \text { COX-2, } \\
\downarrow N F-\kappa B\end{array}$ & $\begin{array}{l}25,50,100 \text { ( } 7 \text { days) } \\
\text { Dose dependant } 100 \\
\text { most effective }\end{array}$ & $\begin{array}{l}\text { Cerebral ischemia/reperfusion, rat (Li et al., } \\
\text { 2019) }\end{array}$ \\
\hline & & $\begin{array}{l}\downarrow \text { lba- } 1, \downarrow \mathrm{IL}-1 \beta, \downarrow \mathrm{IL}-6, \downarrow \text { TNF } \alpha, \downarrow \mathrm{MCP}-1, \downarrow \mathrm{ICAM}-1, \\
\downarrow \mathrm{COX}-2, \downarrow \mathrm{HMGB} 1, \downarrow \text { TLR4 }\end{array}$ & $\begin{array}{l}20 \text { and } 50 \text { ( } 7 \text { days) } \\
\text { Dose dependant } 50 \\
\text { most effective }\end{array}$ & $\begin{array}{l}\text { I.P. LPS, adult male BALB/c mouse (Yang } \\
\text { et al., 2019) }\end{array}$ \\
\hline & & $\begin{array}{l}\downarrow \text { Iba1, } \downarrow \text { IL-1 } \beta, \downarrow I L-6, \downarrow \text { TNF- } \alpha, \downarrow \text { MCP- } 1, \downarrow \text { COX-2, } \\
\downarrow \text { iNOS, } \downarrow \text { HMGB1 }, \downarrow \text { TLR4, } \downarrow \text { MyD88 }\end{array}$ & 25,50, or 100 (7 days) & $\begin{array}{l}\text { I.P. LPS, adult male BALB/c mouse (Cheng } \\
\text { et al., 2018) }\end{array}$ \\
\hline & & $\downarrow T N F-\alpha$ & 10 (21 days) & $\begin{array}{l}\text { Intracerebroventricular injection STZ, OVX } \\
\text { female Wistar rat (Kouhestani et al., 2018) }\end{array}$ \\
\hline & Anti-oxidative & $\uparrow S O D, \uparrow G S H, \downarrow M D A$ & 10 (21 days) & $\begin{array}{l}\text { Intracerebroventricular injection STZ, OVX } \\
\text { female Wistar rat (Kouhestani et al., 2018) }\end{array}$ \\
\hline & & $\downarrow \mathrm{MDA}, \uparrow \mathrm{GPx}, \uparrow \mathrm{CAT}, \uparrow \mathrm{SOD}, \uparrow \mathrm{GSK} 3 \beta-\mathrm{Nrf2}$ & 21 (14 days) & $\begin{array}{l}\text { I.P. Chlorpyrifos daily for } 14 \text { days, 8-week } \\
\text { male albino Wistar rat (Hussein et al., 2018) }\end{array}$ \\
\hline & Bioenergetic & $\uparrow A C h E$ activity & 21 (14 days) & $\begin{array}{l}\text { I.P. Chlorpyrifos daily for } 14 \text { days, 8-week } \\
\text { male albino Wistar rat (Hussein et al., 2018) }\end{array}$ \\
\hline & & $\uparrow T C A$ cycle flux, $\downarrow$ acyl carnitines, $\uparrow N$-acetyl aspartate & $\begin{array}{l}1 \text { (three times: } 1,24 \text {, } \\
48 \mathrm{~h} \text { ) }\end{array}$ & $\begin{array}{l}\text { Traumatic Brain Injury, 23-24-day male } \\
\text { Wistar rat (Chitturi et al., 2019) }\end{array}$ \\
\hline & BBB protection & $\downarrow$ EB leakage, $\downarrow$ MMP-3 & $\begin{array}{l}25,50,100 \text { ( } 7 \text { days) } \\
\text { Dose dependant } 100 \\
\text { most effective }\end{array}$ & $\begin{array}{l}\text { Cerebral ischemia/reperfusion, rat (Li et al., } \\
\text { 2019) }\end{array}$ \\
\hline & & $\begin{array}{l}\downarrow \text { ultrastructure destruction (electron microscope), } \\
\uparrow o c c l u d i n, \uparrow c l a u d i n-1, \uparrow C X-43\end{array}$ & $\begin{array}{l}20 \text { and } 50 \text { ( } 7 \text { days) } \\
\text { Dose dependant } 50 \\
\text { most effective }\end{array}$ & $\begin{array}{l}\text { I.P. LPS, adult male BALB/c mouse (Yang } \\
\text { et al., 2019) }\end{array}$ \\
\hline & & $\downarrow$ EB leakage, $\uparrow$ occludin-1, claudin-1, $\uparrow$ CX43 & 25,50 , or 100 (7 days) & $\begin{array}{l}\text { I.P. LPS, adult male BALB/c mouse (Cheng } \\
\text { et al., 2018) }\end{array}$ \\
\hline
\end{tabular}

(I-IV) function and stability. Disturbance of mitochondria function impairs mitochondrial enzyme bioenergetics, reducing ATP production, while simultaneously leading to substantial increases in ROS production thus feeding into the other disease processes.

In addition to mitochondrial function, implications for acetylcholinesterase activity and thus cholinergic transmission were apparent. Generally, citrus flavonoids led to a reduction in acetylcholinesterase activity, accompanied by subsequent increased acetylcholine levels (Ashafaq et al., 2014; Sachdeva et al., 2014; Chtourou et al., 2015; Javed et al., 2015; Khajevand-Khazaei et al., 2018; Ishola et al., 2019; Haider et al., 2020), however the reverse (increased acetylcholinesterase activity) has was also reported (Chtourou et al., 2014; Shagirtha et al., 2017). Again, these discrepancies likely arise as a result of the different models used (Table 1).

\section{Proteinopathy}

Only a limited amount of preclinical evidence exists to support the beneficial effects of citrus on proteinopathies. This primarily relates to Alzheimer's disease, with reports revealing improvements in Tau phosphorylation (Yang W. et al., 2014;
Okuyama et al., 2018; Zhou et al., 2020), and A $\beta$ deposition (Yang W. et al., 2014; Li et al., 2015; Zhou et al., 2020), however no effect has also been reported (Wang et al., 2014). The mechanistic basis for these changes remains relatively weak, although the glycogen synthase kinase 3 beta (GSK3 $\beta$ ) may be a plausible candidate (Yang W. et al., 2014; Zhou et al., 2020). A reduction of $\alpha$-synuclein, the protein attributed to parkinopathy has also been described (Mani et al., 2018), potentially indicating that the effect extends further than Alzheimer's disease-associated proteins. Higher dosage appears crucial to establish these effects, with $<100 \mathrm{mg} / \mathrm{kg}$ failing to have any impact. Finally, a report in which hesperetin was administered demonstrated restoration of brain proteolytic enzyme levels (Shagirtha et al., 2017), potentially linking citrus flavonoids to lysosomal degradation processes (Aufschnaiter et al., 2017) (Table 1).

\section{Anti-apoptotic}

Ultimately, neurodegeneration which leads to cognitive impairment can be defined as the progressive loss of neurons. Dysregulation of the above-mentioned processes categorically leads to neuronal cell death. Thus, the modulation achieved through citrus flavonoids demonstrates a switch from a 
TABLE 2 | Overview of citrus flavonoids on cognitive performance and locomotor activity in preclinical models.

Citrus polyphenol Behavioral test performance

\section{Hesperidin}

No change locomotor activity (OF), $\downarrow$ anxiety-like behavior (EPM), $\downarrow$ depressive symptoms (splash test)

$\downarrow$ Anxiety-like behavior (EPM, OF), $\downarrow$ Depressive symptoms (FST)

$\downarrow$ Anhedonia (SPT), $\downarrow$ Depressive symptoms (TST and FST), $\downarrow$ Anxiety-like behavior (OF)

$\uparrow$ Recognition memory (NOL and NOR)

$\downarrow$ Anhedonia (SPT), $\downarrow$ Depressive symptoms (TST, FST, Novelty-suppressed feeding)

$\uparrow$ Spatial learning and memory (MWM and $Y$ maze)

$\downarrow$ Depressive symptoms (TST and FST)

$\downarrow$ Anxiety-like behavior (OF), $\downarrow$ Depressive symptoms (FST, rearing, grooming, splash test), $\uparrow$ Spatial learning and memory (MWM), $\uparrow$ Recognition memory (NOR) $\downarrow$ Anhedonia (SPT), $\downarrow$ Depressive symptoms (FST), No change OF

$\downarrow$ Anhedonia (SPT), $\downarrow$ Depressive symptoms (FST)

$\uparrow$ Social behavior (Nest building, resident-intruder assay)

$\uparrow$ Spatial learning and memory (MWM)

$\downarrow$ Depressive symptoms (FST)

$\downarrow$ Abnormal thigmotaxis (OF), $\uparrow$ Recognition memory (NOR), Spatial reference learning and memory (MWM)

$\downarrow$ Depressive symptoms (TST), $\downarrow$ Spatial memory (MWM), No change OF

Hesperetin

$\uparrow$ Spatial learning and memory (MWM and Y-Maze)

$\uparrow$ Recognition memory (NOR), $\uparrow$ Spatial learning and memory (MWM)

$\uparrow$ Recognition memory (NOR), $\uparrow$ Associative memory (PAT)

Naringenin $\uparrow$ Spatial learning and memory (MWM and Barnes maze)

$\uparrow$ Spatial learning and memory (MWM), $\uparrow$ Recognition memory (NOR), $\uparrow$ Associative memory (PAT), $\uparrow$ Working memory (EPM), $\uparrow$ Social behavior, $\downarrow$ Depressive symptoms (FST)

$\uparrow$ Spatial learning and memory (MWM), $\uparrow$ Recognition memory (NOR)

$\downarrow$ Anxiety-like behavior (OF), $\downarrow$ Depressive symptoms (FST), $\uparrow$ Spatial learning and memory (MWM)

$\downarrow$ Anhedonia (SPT), $\downarrow$ Depressive symptoms (TST and FST)

$\uparrow$ Spatial learning and memory (Y-maze, $\uparrow$ Recognition memory (NOR), $\uparrow$ Associative memory (PAT)

$\uparrow$ Motor Function (beam walk, vertical grid, horizontal grid)

$\uparrow$ Spatial learning and memory (MWM)

Reported effective Dose (mg/kg)

50 (28 days)

50, 150 (10 weeks) Dose dependent with 150 more effective

100 and 200 (three times a week for 3 weeks)

100 (21 days)

50 (14 days)

25, 50, 100 (14 days). Dose dependent with 50 and 100 more effective

100 (60 days)

50 (14 days)

25, 50, 100 (7 days)

25, 50 (3 weeks)

100 (10 days)

100 and 200 (15 days)

25, 50, 100 (21 days)

50, 100 (16 weeks) Dose dependent with 100 more effective

50 (28 days)

50 (5 weeks)

5, 50 (3 days)

10, 20 (3 weeks)

250-300 (10 weeks)

50 (14 days)

100 (15 days)

50 (4 weeks)

25, 50, 100 (5 days) Dose dependent with 50 and 100 more effective

50, 100 (7 days)

$25,50,100$ (5 days)

50 (16 days)
Model summary/References

Intrastriatal injection 6-OHD, 3-6-month adult male C57BL/6 mouse (Antunes et al., 2020)

I.P injection STZ, adult male Sprague Dawley rat (Zhu et al., 2020)

Chronic unpredictable mild stress, 6-8-week ICR male mouse (Fu et al., 2019)

IV injection Methotrexate, male Sprague Dawley rat (Naewla et al., 2019)

Concussive head injury, 10-11-week male NMRI mouse (Kosari-Nasab et al., 2018)

L-Methionine treated, male Wistar rat (Hemanth

Kumar et al., 2017)

I.P. AlCl3, 10-12-week male albino Wistar rat (Justin Thenmozhi et al., 2017)

Olfactory bulbectomy, male C57BL/6 mouse

(Antunes et al., 2016)

I.P. LPS, male ICR mouse (Li M. et al., 2016)

Chronic mild stress, 5-week male ICR mouse (Li C. F. et al., 2016)

Transgenic APP/PS1-21, 5-month male mouse (Li et al., 2015)

Intracerebroventricular injection STZ, 12-month Swiss male albino mouse (Javed et al., 2015) I.P. STZ, male albino Wistar rat (El-Marasy et al., 2014)

APPswe/PS1dE9, 3-month male mouse (Wang et al., 2014)

Intracerebroventricular injection 6-OHDA, female C57 BL/6 mouse (Antunes et al., 2014)

I.P. LPS, 7-8-week male C57BL/6N mouse (Muhammad et al., 2019)

I.P. scopolamine, male albino mouse (Ishola et al., 2019)

I.P. STZ male Wistar rat (Kheradmand et al., 2018) High-fat diet-fed, 10-month SAMP8 male mouse (Zhou et al., 2020)

I.P. $\mathrm{AlCl}_{3}+$ D-gal injection, young albino Wistar rat (Haider et al., 2020)

Oral MeHg, 5-week male Swiss albino mouse (Krishna Chandran et al., 2019)

Chronic unpredictable mild stress, adult male Wistar rat (Tayyab et al., 2019)

Bilateral olfactory bulbectomy, 3-month BALB/c male mouse (Bansal et al., 2018)

I.P. LPS male albino Wistar rat (Khajevand-Khazaei et al., 2018)

I.P. injection MPTP, male C57BL/6J mouse (Mani et al., 2018)

Young adult male albino Wistar rat (Liaquat et al., 2018) 
TABLE 2 | Continued

\begin{tabular}{|c|c|c|c|}
\hline Citrus polyphenol & Behavioral test performance & $\begin{array}{l}\text { Reported effective Dose } \\
\text { (mg/kg) }\end{array}$ & Model summary/References \\
\hline & $\begin{array}{l}\uparrow \text { Spatial learning and memory (Y-maze, RAM), } \uparrow \text { Associative } \\
\text { memory (PAT) }\end{array}$ & 100 (single dose) & $\begin{array}{l}\text { Dorsal hippocampal } A \beta \text { injection, adult male Wistar } \\
\text { rat (Ghofrani et al., 2015) }\end{array}$ \\
\hline & $\begin{array}{l}\downarrow \text { Anhedonia (SPT), } \downarrow \text { Anxiety (Novelty-suppressed feeding } \\
\text { test) }\end{array}$ & 10, 20 (3 weeks) & $\begin{array}{l}\text { Chronic unpredictable mild stress, 5-week male } \\
\text { ICR mouse (Yi et al., 2014) }\end{array}$ \\
\hline & $\uparrow$ Spatial learning and memory (MWM) & $25,50,100$ (21 days) & $\begin{array}{l}\text { Intracerebroventricular injection, STZ adult male } \\
\text { Sprague Dawley rat (Yang W. et al., 2014) }\end{array}$ \\
\hline & $\begin{array}{l}\uparrow \text { Motor Function (Rota rod, Grip test, Adhesive-removal } \\
\text { test) }\end{array}$ & 50 (21 days) & $\begin{array}{l}\text { Middle cerebral artery occlusion, 16-week male } \\
\text { Wistar rat (Raza et al., 2013) }\end{array}$ \\
\hline \multirow[t]{6}{*}{ Naringin } & $\begin{array}{l}\uparrow \text { Locomotor activity (Rotarod, Beam-crossing, footprint } \\
\text { analyses), } \downarrow \text { Depressive symptoms (Grooming) }\end{array}$ & 40 and $80 \uparrow(28$ days) & $\begin{array}{l}\text { Intrastriatal injection quinolinic acid, adult male } \\
\text { Sprague-Dawley rat (Cui et al., 2018) }\end{array}$ \\
\hline & $\begin{array}{l}\uparrow \text { Motor/locomotor activity (Horizontal Bar, Spontaneous } \\
\text { Motility, Forelimb Flexion, Righting Reflex, Actophotometer, } \\
\text { Rotarod, Paw withdrawal) } \downarrow \text { Depressive symptoms (FST) } \\
\uparrow S p a t i a l \text { learning and memory (MWM) }\end{array}$ & $\begin{array}{l}20 \text { and } 40 \text { ( } 28 \text { days) Dose } \\
\text { dependent } 40 \text { most } \\
\text { effective }\end{array}$ & $\begin{array}{l}\text { Intastriatal injection collagenase, adult female } \\
\text { Wistar rat (Singh et al., 2017) }\end{array}$ \\
\hline & $\begin{array}{l}\downarrow \text { Motor abnormalities (Hind limb function test, Grip strength } \\
\text { test, Print length analysis) }\end{array}$ & 80 (14 days) & $\begin{array}{l}\text { I.P. 3-nitropropionic acid, male Wistar rat (Gopinath } \\
\text { and Sudhandiran, 2016) }\end{array}$ \\
\hline & $\begin{array}{l}\uparrow \text { Spatial learning and memory (MWM), } \uparrow \text { Recognition } \\
\text { memory (NOR) }\end{array}$ & 100 (20 weeks) & $\begin{array}{l}\text { High-fat diet induced cognitive decline, male } \\
\text { C57BL/6 mouse (Wang et al., 2015b) }\end{array}$ \\
\hline & $\downarrow$ Anxiety-like behavior (OF, elevated T maze) & $\begin{array}{l}\text { 25, 50, } 100 \text { ( } 5 \text { weeks) Dose } \\
\text { dependent with } 100 \text { most } \\
\text { effective }\end{array}$ & $\begin{array}{l}\text { Cisplatin exposed, middle aged Wistar rat } \\
\text { (Chtourou et al., 2015) }\end{array}$ \\
\hline & $\begin{array}{l}\uparrow S p a t i a l \text { learning and memory (MWM) } \text { (Working memory } \\
\text { (EPM) }\end{array}$ & 50,100, and 200 ( 21 days) & $\begin{array}{l}\text { Intracerebroventricular STZ, adult male Wistar rats } \\
\text { (Sachdeva et al., 2014) }\end{array}$ \\
\hline \multirow[t]{3}{*}{ Kaempferol } & $\begin{array}{l}\uparrow \text { Sensorimotor behaviors (Whisker stimulation-induced } \\
\text { motor response, Forelimb usage test) }\end{array}$ & 1 (three times: $1,24,48$ h) & $\begin{array}{l}\text { Traumatic Brain Injury, 23-24-day male } \\
\text { Sprague-Dawley rat (Chitturi et al., 2019) }\end{array}$ \\
\hline & $\begin{array}{l}\uparrow S p a t i a l \text { learning and memory (Y-maze), } \uparrow \text { Recognition } \\
\text { memory (NOR) }\end{array}$ & 21 (14 days) & $\begin{array}{l}\text { I.P. Chlorpyrifos daily for } 14 \text { days, 8-week male } \\
\text { albino Wistar rat (Hussein et al., 2018) }\end{array}$ \\
\hline & $\uparrow$ Spatial learning and memory (MWM) & 10 (21 days) & $\begin{array}{l}\text { Intracerebroventricular injection STZ, OVX female } \\
\text { Wistar rats (Kouhestani et al., 2018) }\end{array}$ \\
\hline
\end{tabular}

disease state to an overall anti-apoptotic environment and in some cases an additional increase proliferation/neurogenesis. Amongst markers of these effects, the nuclear protein Ki67 and the microtubule-associated protein doublecortin (DCX) were reported in addition to the apoptotic regulating proteins Bax-Bcl2, and ERK-BDNF (Table 1).

\section{Cognitive Outcomes}

Unsurprisingly, given the established effects of flavonoids on key markers of brain health and functionality at molecular and metabolic levels, in studies where cognition was determined, flavonoid supplementation led to improvements in cognitive performance. Cognitive and behavioral assessment further supports/demonstrates the neuroprotective properties associated with citrus flavonoids (Table 2). Anxiolytic and antidepressant actions were particularly prominent across the literature and appeared to be consistent across several disease models, suggesting modulation of a fundamental anxiety and depression related process. Similarly, citrus polyphenol supplementation improved deficits in learning and memory, specifically spatial and recognition memory, which may indicate protection of medial temporal lobe, particularly vulnerable to Alzheimer's like neurodegenerative diseases. Improvements in motor functions and locomotion were also apparent, and impressively remained even in most severe models such as middle cerebral artery occlusion (Raza et al., 2013), and traumatic brain injury (Chitturi et al., 2019).

\section{HUMAN EVIDENCE FOR CITRUS POLYPHENOLS AND BRAIN HEALTH}

Whilst a wealth of pre-clinical (reviewed above) and in vitro data exploring neurocognitive outcomes in relation to citrus flavonoids is currently available, the same cannot be said for human trials which remain considerably limited in number. In our literature search, we identified 10 human studies (five observational; five interventions) assessing the effects of citrus flavonoids on brain health and cognition in healthy adults, or in addition to other co-morbidities including depression, dementia, schizophrenia, and stroke.

\section{Studies on Healthy Adults}

The benefits of citrus fruit consumption in the context of healthy aging were highlighted by a cross-sectional study involving 2031 elderly (aged 70-74 years) Norwegian individuals which explored the impact of different plant foods on cognitive performance (Nurk et al., 2010). Study participants underwent extensive cognitive testing in addition to completing comprehensive food frequency questionnaires. After adjustment for multiple 
TABLE 3 | Overview of intervention studies on citrus fruits in brain health and disease in humans.

\begin{tabular}{|c|c|c|c|c|c|}
\hline Study & Study design & $\begin{array}{l}\text { Participants who } \\
\text { completed study }\end{array}$ & Disease/state & $\begin{array}{l}\text { Intervention or } \\
\text { variable of interest }\end{array}$ & Duration \\
\hline Lamport et al., 2016 & $\begin{array}{l}\text { Single blind } \\
\text { randomized trial }\end{array}$ & $\begin{array}{l}40 \text { healthy adults } \\
\text { (18-30 years) }\end{array}$ & N/A (Healthy adults) & $\begin{array}{l}\text { High flavanone drink } \\
(70.5 \mathrm{mg})\end{array}$ & Acute \\
\hline Alharbi et al., 2016 & $\begin{array}{l}\text { Double blind } \\
\text { randomized trial }\end{array}$ & $\begin{array}{l}24 \text { healthy males } \\
\text { (30-65 years) }\end{array}$ & N/A (Healthy adults) & $\begin{array}{l}\text { Flavonoid rich orange } \\
\text { juice ( } 272 \mathrm{mg})\end{array}$ & Acute \\
\hline Kean et al., 2015 & $\begin{array}{l}\text { Double blind } \\
\text { randomized control trial }\end{array}$ & $\begin{array}{l}37 \text { healthy adults } \\
\text { (60-81 years) }\end{array}$ & N/A (Healthy adults) & $\begin{array}{l}\text { High flavanone } 100 \% \\
\text { orange juice (305 mg) }\end{array}$ & 8 weeks \\
\hline Park et al., 2020 & $\begin{array}{l}\text { Randomized single } \\
\text { blind study }\end{array}$ & 40 adults (20-30 years) & $\begin{array}{l}\text { Depressive symptoms } \\
\text { and microbiota }\end{array}$ & $\begin{array}{l}\text { Flavonoid rich orange } \\
\text { juice }(600 \mathrm{mg})\end{array}$ & 8 weeks \\
\hline Chang et al., 2016 & $\begin{array}{l}\text { Prospective cohort } \\
\text { study }\end{array}$ & $\begin{array}{l}82,643 \text { females }(36-55 \\
\text { and } 53-80 \text { years) }\end{array}$ & Depression & $\begin{array}{l}\text { Total dietary flavonoid } \\
\text { intake (including } \\
\text { flavanones) }\end{array}$ & 10 year follow up \\
\hline Zhang et al., 2017 & $\begin{array}{l}\text { Retrospective cohort } \\
\text { study }\end{array}$ & $\begin{array}{l}13,373 \text { adults } \\
(\geq 65 \text { years) }\end{array}$ & Dementia & Daily citrus intake & 5.7 year follow up \\
\hline Bruno et al., 2017 & Open label pilot study & 20 adults (age n/a) & $\begin{array}{l}\text { Cognitive dysfunction in } \\
\text { Schizophrenia }\end{array}$ & $\begin{array}{l}\text { Bergamot polyphenolic } \\
\text { fraction ( } 1000 \text { mg/day) }\end{array}$ & 8 weeks \\
\hline Nurk et al., 2010 & Cross-sectional study & $\begin{array}{l}2031 \text { Elderly adults } \\
\text { (70-74 years) }\end{array}$ & Cognitive decline & $\begin{array}{l}\text { Different plant foods } \\
\text { (including citrus intake) }\end{array}$ & $\mathrm{N} / \mathrm{A}$ \\
\hline Goetz et al., 2016 & $\begin{array}{l}\text { Biracial prospective } \\
\text { study }\end{array}$ & $\begin{array}{l}\text { 20,024 participants } \\
\text { (>45 years) }\end{array}$ & Stroke incident & $\begin{array}{l}\text { Different flavonoid } \\
\text { intake (including } \\
\text { flavanones) }\end{array}$ & 6.5 years follow up \\
\hline Cassidy et al., 2012 & $\begin{array}{l}\text { Prospective cohort } \\
\text { study }\end{array}$ & $\begin{array}{l}\text { 69,622 females } \\
\text { (30-55 years) }\end{array}$ & Stroke incident & $\begin{array}{l}\text { Dietary flavonoid intake } \\
\text { (including flavanones) }\end{array}$ & 14 years follow up \\
\hline
\end{tabular}

testing, citrus fruits had the strongest association with cognitive test performance. Kendrick object learning, trial making, digit symbol and block design tasks all showed statistically significant improvements suggestive of better episodic memory, executive function, perceptual speed, and visuospatial skills (Table 3).

Following on from these observations, Kean et al. (2015), examined the chronic consumption of flavanone-rich orange juice in relation to cognition in 37 healthy volunteers (6081 years). The study was a double-blind RCT with a crossover design and involved consumption of a $500 \mathrm{~mL}$ high flavanone (daily serving of $305 \mathrm{mg}$ - $549 \mathrm{mg}$ hesperidin/L and $60 \mathrm{mg}$ narirutin/L) orange juice daily for 8 weeks. In support of the cross-sectional study mentioned above, global cognition increased in response to chronic consumption of the flavanonerich orange juice relative to control. In addition, high flavanone intake significantly improved recall and nominally increased executive function, although post hoc analysis found this to be non-significant $(p=0.06)$. These effects were independent of mood and blood pressure which both remained unchanged (Table 3).

Acute neurological responses to citrus flavonoids have similarly been investigated (Alharbi et al., 2016; Lamport et al., 2016). Firstly, in a randomized, double-blind, placebo-controlled, crossover trial, Alharbi et al. (2016) explored the cognitive benefits associated with flavonoid-rich orange juice $(272 \mathrm{mg}-220.46 \mathrm{mg}$ hesperidin, $34.54 \mathrm{mg}$ narirutin, $17.14 \mathrm{mg}$ other flavonoids) at 2 and $6 \mathrm{~h}$ post-consumption in 24 healthy middle-aged adults (30-65 years). From the cognitive battery performed, flavonoid rich orange juice consumption led to higher performance in Simple Finger Tapping (measure of psychomotor speed) and Continuous Performance Task (measure of attention and more broadly executive function) at 2 and $6 \mathrm{~h}$ respectively. A non-significant trend for higher global cognitive performance (all tests combined) was also observed, as well as an increase in subjective alertness. Interestingly, significant improvements observed in cognition and subjective alertness at $6 \mathrm{~h}$ coincide with an anticipated peak in flavanone metabolites at 5-7 h (Manach et al., 2003), although this was not physically measured.

As with the previous study, Lamport et al. (2016) assessed acute neurological response, this time to a commercially available high flavanone beverage ( $\mathrm{HF}, 70.5 \mathrm{mg}-42.15 \mathrm{mg}$ hesperidin, $17.25 \mathrm{mg}$ naringin, $6.75 \mathrm{mg}$ narirutin, $4.3 \mathrm{mg}$ caffeic acid) and utilizing an additional measure of cerebral blood flow (CBF). The study was single blind, randomized, cross over, by design involving healthy adult volunteers (aged 1830 years). Participants underwent either cognitive testing $2 \mathrm{~h}$ post consumption $(n=28)$ or completed an fMRI assessment of CBF 2 and 5 h post consumption $(n=16)$. High flavanone beverage intake significantly increased cerebral perfusion in the inferior frontal and middle right frontal gyrus in the right hemisphere at $2 \mathrm{~h}$. Similarly, at $2 \mathrm{~h}$, improvement in digit symbol substitution test (a measure of executive function) was seen, correlating with the increased regional perfusion of the inferior frontal gyrus, known to be involved in executive function (Aron et al., 2004). Despite the extensive cognitive battery, no additional effects were found. However, as addressed by the authors, this study had a number of limitations. In particular, the fact MRI and cognitive test were performed on separate individuals, and that cognitive tests were only performed at $2 \mathrm{~h}$ limits comparative potential. Additionally, as stated by the authors, the fact that the participants were generally young and 
highly educated may have limited the response given they were likely to be optimally functioning (Table 3).

\section{Studies on Depression}

Depression is a complex mood disorder which can often be challenging to treat effectively. Preclinical studies have reported anti-depressant effects of flavonoids, usually attributable to their antioxidant and anti-inflammatory characteristics, and inhibition of monoamine oxidases (Hritcu et al., 2017). An important serological marker identified in depression is BDNF (involved in processes within the central nervous system) which has been observed to be significantly lower in patients with major depressive disorder compared to non-depressed control groups, and subsequently recovered antidepressant users (Chen et al., 2001). Interestingly, BDNF is often increased in response to flavonoid consumption (Neshatdoust et al., 2016).

In a prospective cohort study following 82,643 women with no previous diagnosis of depression from the Nurses' Health Study (NHS; aged 53-80 years) and Nurses' Health Study II (NHSII; aged 36-55 years) throughout a 10-year period, an inverse association between incident depression and citrus intake, with greatest flavanone intake (>64.2 mg/day) resulting in a significant $10 \%$ reduction in incident depression risk was observed. Furthermore, citrus fruit and juice intake of $>2$ servings/day had HR of 0.82 (Chang et al., 2016).

These anti-depressant effects of citrus flavonoids were recently put to the test by Park et al. (2020), in a single blind, randomized control study examining the effects of daily $380 \mathrm{ml}$ flavonoid rich (FR; $600 \pm 5.4 \mathrm{mg}$ flavonoid content) orange juice consumption on depressive symptoms and gut microbiota for an 8-week period in young adults (aged 20-30 years). The results in relation to depression were by no means clear cut, with no apparent significant differences between high and low flavonoid groups after 8 weeks. However, compared with baseline the results suggested potential improvement in BDNF, and to some extent to the Center for Epidemiological Studies Depression Scale (CES-D), a psychiatric screening tool used to detect pre-existing mental disorders, although both treatments appeared to improve upon baseline CES-D scores (Table 3).

\section{Studies on Dementia}

Despite the preclinical evidence backing the beneficial effect of citrus flavonoids in models of Alzheimer's and Parkinson's diseases, etc. there remains very limited evaluation at the human level.

In a retrospective cohort study (Zhang et al., 2017), examined the association between daily citrus intake and dementia incidence in 13,373 participants (age $\geq 65$ years). FFQs in combination with the Japanese Long-term Care Insurance database were used over a 5.7 years follow up period. Overall, an inverse dose-response relationship was established between weekly citrus fruit intake and incident dementia, with hazard ratios for citrus fruit consumption 3-4 times/week and every day $0.92(95 \%$ CI $0.80,1.07)$ and $0.77(95 \%$ CI $0.73,1.01)$ respectively. As dementia represents a wide range of neurodegenerative diseases, lack of further classification limits the extent to which we can attribute these effects to specific neurodegenerative diseases and should be considered in future studies (Table 3 ).

\section{Studies on Schizophrenia}

Schizophrenia is a complex psychotic condition affecting cognition, the cognitive hallmark of schizophrenia being poor learning and retention of verbal information (Bowie and Harvey, 2006). In an open label pilot study, 20 outpatients diagnosed with schizophrenia receiving second generation antipsychotic medication consumed a flavanone-rich bergamot polyphenolic fraction (BPF) daily at a dose of $1000 \mathrm{mg} /$ day for 8 weeks. Daily BPF consumption significantly improved Wisconsin Card Sorting Test "perseverative errors" and semantic fluency test. With a trend for other cognitive variables evident (Bruno et al., 2017) (Table 3).

\section{Studies on Stroke and Stroke Risk Factors}

The protective effects of citrus flavonoids in cerebrovascular disease are well documented (Testai et al., 2017; Mahmoud et al., 2019) and appears to extend to stroke incidence. A prospective cohort study, assessing the association of dietary flavonoid intake toward stroke risk, followed 69,622 women (30-55 years) from the NHS Study throughout a 14-year period (Cassidy et al., 2012). Although flavonoid intake was not inversely associated with stroke risk, women with greatest intake of flavanones > $62.95 \mathrm{mg} /$ day had a relative risk for experiencing an ischemic stroke of 0.81 compared to lowest intake $<13.72 \mathrm{mg} /$ day. Additionally, an inverse trend was established between citrus fruit/juice intake and risk of ischemic stroke. These apparently protective effects did not influence haemorrhagic strokes (Scheffers et al., 2018; D'Elia et al., 2020; Zurbau et al., 2020).

A recent prospective cohort study (Goetz et al., 2016), utilizing 'The Reasons for Geographic and Racial Differences in Stroke' (REGARDS) database, assessed whether any association exists between flavonoid intake and incident ischemic stroke in a biracial cohort. 20,024 participants ( $>45$ years) of whom flavonoid consumption was determined from FFQ, were followed for 6.5 years. After multivariable adjustment, the highest flavanone intake $>48 \mathrm{mg} /$ day was inversely associated with incidences of ischemic stroke compared to lowest flavanone intake $<3.9 \mathrm{mg} /$ day. With citrus fruit/juice consumption displaying similarly reduced risk (HR of 0.69). In agreement with the above-mentioned study of total flavonoids and other flavonoid subclasses did not show any statistically significant association with incident ischemic stroke, clearly showcasing the importance of the flavanone subclass (Table 3).

Of particular relevance to the mitigation of stroke risk, and also neurodegenerative conditions, it is worth briefly mentioning the influence of citrus flavonoid intake on blood pressure, and vascular functions. RCTs in which flavonoids were administered in the form of orange juice have established significant reductions 
in blood pressure (Morand et al., 2011; Valls et al., 2020), pulse pressure (Valls et al., 2020), while also improving flow-mediated dilation (Li et al., 2020).

\section{FUTURE PERSPECTIVES AND CONCLUSION}

The preclinical literature search distinguished fundamental mechanisms central to citrus flavonoids, with protective effects linked with anti-oxidative and anti-inflammatory action particularly well established. Yet, there are a number of areas requiring further investigation. First, the overwhelming majority of studies to date involve relatively young male animals making it difficult to establish whether or not the observable effects are sex or age specific. Nor do we have an understanding of modulation by either factor. Second, the models employed tend to lean toward the severe side of the disease spectrum, thus translation to a healthy aging context, or milder conditions, remains to be fully determined, although some initial work provides supportive results (Liaquat et al., 2018). Third, despite recent attention on the relationship between gut microbiota and polyphenols, and the apparent ability of citrus flavonoids to mitigate LPS associated neuroinflammation/NDG, to our knowledge there are no studies which have explored the role of the microbiota in combination with citrus flavonoids in the context of brain health and disease. Finally, as can be seen in Table 1, the impact of citrus flavonoids upon BBB integrity/function appears to have emerged over the last couple of years (Cheng et al., 2018; Li et al., 2019; Yang et al., 2019; Lee et al., 2020), particularly for kaempferol, and therefore should be examined in further studies, especially as BBB integrity has similarly been associated with gut microbiota (Braniste et al., 2014).

\section{REFERENCES}

Agosta, F., Caso, F., and Filippi, M. (2013). Dementia and neuroimaging. J. Neurol. 260, 685-691. doi: 10.1007/s00415-012-6778-x

Alharbi, M. H., Lamport, D. J., Dodd, G. F., Saunders, C., Harkness, L., Butler, L. T., et al. (2016). Flavonoid-rich orange juice is associated with acute improvements in cognitive function in healthy middleaged males. Eur. J. Nutr. 55, 2021-2029. doi: 10.1007/s00394-0151016-9

Antunes, M. S., Cattelan Souza, L., Ladd, F. V. L., Ladd, A., Moreira, A. L., Bortolotto, V. C., et al. (2020). Hesperidin ameliorates anxiety-depressivelike behavior in 6-OHDA model of Parkinson's disease by regulating striatal cytokine and neurotrophic factors levels and dopaminergic innervation loss in the striatum of mice. Mol. Neurobiol. 57, 3027-3041. doi: 10.1007/s12035-02001940-3

Antunes, M. S., Goes, A. T., Boeira, S. P., Prigol, M., and Jesse, C. R. (2014). Protective effect of hesperidin in a model of Parkinson's disease induced by 6-hydroxydopamine in aged mice. Nutrition 30, 1415-1422. doi: 10.1016/j.nut.2014. 03.024

Antunes, M. S., Jesse, C. R., Ruff, J. R., de Oliveira Espinosa, D., Gomes, N. S., Altvater, E. E. T., et al. (2016). Hesperidin reverses cognitive and depressive disturbances induced by olfactory bulbectomy in mice by modulating hippocampal neurotrophins and cytokine levels and acetylcholinesterase activity. Eur. J. Pharmacol. 789, 411-420. doi: 10.1016/j.ejphar.2016. 07.042
From a human perspective, there is an obvious lack of human clinical studies which needs to be addressed if a robust assessment of therapeutic potential is to be made. Similarly, very few human studies have followed up on the mechanistic insights established in the preclinical setting. Future human studies should take note of the limitations arising from other human studies in which nutraceuticals were assessed in the context of brain health and disease, for example ensuring optimal participant targeting (in demographics where significant change is most likely to occur), dosage, timing, and duration of treatment for measurable effects to be established.

The complex mixtures of polyphenols present in citrus fruits and juices and their bioactive nuances likely convey greater benefit than one purified compound, accumulatively acting upon multiple targets, and producing synergistic effects. Given the multifactorial nature of neurodegenerative diseases, one would speculate that this complex form would therefore offer greater efficacy, but this is yet to be fully determined.

In conclusion, although significant work remains to fully establish the benefits of citrus polyphenols in brain health and disease, the accumulating in vitro and preclinical data combined with the support of steadily emerging human studies indicates future potential.

\section{AUTHOR CONTRIBUTIONS}

MP, MMM, and DV wrote the manuscript. MP, MMM, EC, $\mathrm{MM}$, and DV contributed to the literature search and edited the manuscript. All the authors contributed to the article and approved the submitted version.

Aron, A. R., Robbins, T. W., and Poldrack, R. A. (2004). Inhibition and the right inferior frontal cortex. Trends Cogn. Sci. 8, 170-177. doi: 10.1016/j.tics.2004. 02.010

Aschoff, J. K., Riedl, K. M., Cooperstone, J. L., Högel, J., Bosy-Westphal, A., Schwartz, S. J., et al. (2016). Urinary excretion ofCitrusflavanones and their major catabolites after consumption of fresh oranges and pasteurized orange juice: a randomized cross-over study. Mol. Nutr. Food Res. 60, 2602-2610. doi: 10.1002/mnfr.201600315

Ashafaq, M., Varshney, L., Khan, M. H., Salman, M., Naseem, M., Wajid, S., et al. (2014). Neuromodulatory effects of hesperidin in mitigating oxidative stress in streptozotocin induced diabetes. Biomed. Res. Int. 2014:249031. doi: 10.1155/ 2014/249031

Aufschnaiter, A., Kohler, V., and Büttner, S. (2017). Taking out the garbage: cathepsin D and calcineurin in neurodegeneration. Neural Regen. Res. 12, 1776-1779. doi: 10.4103/1673-5374.219031

Bai, X., Zhang, X., Chen, L., Zhang, J., Zhang, L., Zhao, X., et al. (2014). Protective effect of naringenin in experimental ischemic stroke: down-regulated NOD2, RIP2, NF-кB, MMP-9 and up-regulated claudin-5 expression. Neurochem. Res. 39, 1405-1415. doi: 10.1007/s11064-014-1326-y

Bansal, Y., Singh, R., Saroj, P., Sodhi, R. K., and Kuhad, A. (2018). Naringenin protects against oxido-inflammatory aberrations and altered tryptophan metabolism in olfactory bulbectomized-mice model of depression. Toxicol. Appl. Pharmacol. 355, 257-268. doi: 10.1016/j.taap.2018.07.010

Barrientos, R. M., Kitt, M. M., Watkins, L. R., and Maier, S. F. (2015). Neuroinflammation in the normal aging hippocampus. Neuroscience 309, 84 99. doi: 10.1016/j.neuroscience.2015.03.007 
Bellavite, P., and Donzelli, A. (2020). Hesperidin and SARS-CoV-2: new light on the healthy function of citrus fruits. Antioxidants 9:742. doi: 10.3390/ antiox 9080742

Bowie, C. R., and Harvey, P. D. (2006). Cognitive deficits and functional outcome in schizophrenia. Neuropsychiatr. Dis. Treat. 2, 531-536. doi: 10.2147/nedt.2006.2. 4.531

Brai, E., Hummel, T., and Alberi, L. (2020). Smell, an underrated early biomarker for brain aging. Front. Neurosci. 14:792. doi: 10.3389/fnins.2020.00792

Braniste, V., Al-Asmakh, M., Kowal, C., Anuar, F., Abbaspour, A., Toth, M., et al. (2014). The gut microbiota influences blood-brain barrier permeability in mice. Sci. Transl. Med. 6, ra158-ra263. doi: 10.1126/scitranslmed.3009759

Brasili, E., Hassimotto, N. M. A., Del Chierico, F., Marini, F., Quagliariello, A., Sciubba, F., et al. (2019). Daily consumption of orange juice from Citrus sinensis L. Osbeck cv. Cara Cara and cv. Bahia differently affects gut microbiota profiling as unveiled by an integrated meta-omics approach. J. Agric. Food Chem. 67, 1381-1391. doi: 10.1021/acs.jafc. 8 b05408

Brett, G. M., Hollands, W., Needs, P. W., Teucher, B., Dainty, J. R., Davis, B. D., et al. (2008). Absorption, metabolism and excretion of flavanones from single portions of orange fruit and juice and effects of anthropometric variables and contraceptive pill use on flavanone excretion. Br. J. Nutr. 101, 664-675. doi: $10.1017 /$ s000711450803081x

Bruno, A., Pandolfo, G., Crucitti, M., Cedro, C., Zoccali, R. A., and Muscatello, M. R. A. (2017). Bergamot polyphenolic fraction supplementation improves cognitive functioning in schizophrenia: data from an 8-week, openlabel pilot study. J. Clin. Psychopharmacol. 37, 468-471. doi: 10.1097/jcp. 0000000000000730

Camandola, S., and Mattson, M. P. (2017). Brain metabolism in health, aging, and neurodegeneration. EMBO J. 36, 1474-1492. doi: 10.15252/embj.201695810

Cassidy, A., Rimm, E. B., O'Reilly, E. J., Logroscino, G., Kay, C., Chiuve, S. E., et al. (2012). Dietary flavonoids and risk of stroke in women. Stroke 43, 946-951. doi: 10.1161/strokeaha.111.637835

Catalkaya, G., Venema, K., Lucini, L., Rocchetti, G., Delmas, D., Daglia, M., et al. (2020). Interaction of dietary polyphenols and gut microbiota: microbial metabolism of polyphenols, influence on the gut microbiota, and implications on host health. Food Front. 1, 109-133. doi: 10.1002/fft2.25

Chandra, A., Valkimadi, P. E., Pagano, G., Cousins, O., Dervenoulas, G., and Politis, M. (2019). Applications of amyloid, tau, and neuroinflammation PET imaging to Alzheimer's disease and mild cognitive impairment. Hum. Brain Mapp. 40, 5424-5442. doi: 10.1002/hbm.24782

Chang, S. C., Cassidy, A., Willett, W. C., Rimm, E. B., O’Reilly, E. J., and Okereke, O. I. (2016). Dietary flavonoid intake and risk of incident depression in midlife and older women. Am. J. Clin. Nutr. 104, 704-714. doi: 10.3945/ajcn.115. 124545

Chen, B., Dowlatshahi, D., MacQueen, G. M., Wang, J. F., and Young, L. T. (2001). Increased hippocampal BDNF immunoreactivity in subjects treated with antidepressant medication. Biol. Psychiatry 50, 260-265. doi: 10.1016/ s0006-3223(01)01083-6

Cheng, X., Yang, Y. L., Yang, H., Wang, Y. H., and Du, G. H. (2018). Kaempferol alleviates LPS-induced neuroinflammation and BBB dysfunction in mice via inhibiting HMGB1 release and down-regulating TLR4/MyD88 pathway. Int. Immunopharmacol. 56, 29-35. doi: 10.1016/j.intimp.2018. 01.002

Chitturi, J., Santhakumar, V., and Kannurpatti, S. S. (2019). Beneficial effects of kaempferol after developmental traumatic brain injury is through protection of mitochondrial function, oxidative metabolism, and neural viability. J. Neurotrauma 36, 1264-1278. doi: 10.1089/neu.2018.6100

Chiva-Blanch, G., and Badimon, L. (2017). Effects of polyphenol intake on metabolic syndrome: current evidences from human trials. Oxid. Med. Cell Longev. 2017:5812401. doi: 10.1155/2017/5812401

Chtourou, Y., Fetoui, H., and Gdoura, R. (2014). Protective effects of naringenin on iron-overload-induced cerebral cortex neurotoxicity correlated with oxidative stress. Biol. Trace Elem. Res. 158, 376-383. doi: 10.1007/s12011-014-9948-0

Chtourou, Y., Gargouri, B., Kebieche, M., and Fetoui, H. (2015). Naringin abrogates cisplatin-induced cognitive deficits and cholinergic dysfunction through the down-regulation of AChE expression and iNOS signaling pathways in hippocampus of aged rats. J. Mol. Neurosci. 56, 349-362. doi: 10.1007/ s12031-015-0547-0
Contreras, J. A., Aslanyan, V., Sweeney, M. D., Sanders, L. M. J., Sagare, A. P., Zlokovic, B. V., et al. (2020). Functional connectivity among brain regions affected in Alzheimer's disease is associated with CSF TNF- $\alpha$ in APOE4 carriers. Neurobiol. Aging 86, 112-122. doi: 10.1016/j.neurobiolaging.2019.10.013

Coutrot, A., Schmidt, S., Coutrot, L., Pittman, J., Hong, L., Wiener, J. M., et al. (2019). Virtual navigation tested on a mobile app is predictive of real-world wayfinding navigation performance. PLoS One 14:e0213272. doi: 10.1371/ journal.pone.0213272

Cui, J., Wang, G., Kandhare, A. D., Mukherjee-Kandhare, A. A., and Bodhankar, S. L. (2018). Neuroprotective effect of naringin, a flavone glycoside in quinolinic acid-induced neurotoxicity: possible role of PPAR- $\gamma, \mathrm{Bax} / \mathrm{Bcl}-2$, and caspase- 3 . Food Chem. Toxicol. 121, 95-108. doi: 10.1016/j.fct.2018.08.028

Cunnane, S. C., Courchesne-Loyer, A., Vandenberghe, C., St-Pierre, V., Fortier, M., Hennebelle, M., et al. (2016). Can ketones help rescue brain fuel supply in later life? Implications for cognitive health during aging and the treatment of Alzheimer's disease. Front. Mol. Neurosci. 9:53. doi: 10.3389/fnmol.2016. 00053

Cutrim, C. S., and Cortez, M. A. S. (2018). A review on polyphenols: classification, beneficial effects and their application in dairy products. Int. J. Dairy Technol. 71, 564-578. doi: 10.1111/1471-0307.12515

Dalile, B., Van Oudenhove, L., Vervliet, B., and Verbeke, K. (2019). The role of short-chain fatty acids in microbiota-gut-brain communication. Nat. Rev. Gastroenterol. Hepatol. 16, 461-478. doi: 10.1038/s41575-019-0157-3

De Rycker, J., Ruxton, C., Jungen, M., and Schweiggert, R. (2020). Nutritional composition of commercially-produced $100 \%$ orange juice reveals large variability in vascular health bioactive, hesperidin. Proc. Nutr. Soc. 79:E320. doi: $10.1017 / \mathrm{s} 0029665120002682$

D’Elia, L., Dinu, M., Sofi, F., Volpe, M., and Strazzullo, P. (2020). 100\% Fruit juice intake and cardiovascular risk: a systematic review and meta-analysis of prospective and randomised controlled studies. Eur. J. Nutr. doi: 10.1007/ s00394-020-02426-7

Di Meo, F., Lemaur, V., Cornil, J., Lazzaroni, R., Duroux, J.-L., Olivier, Y., et al. (2013). Free radical scavenging by natural polyphenols: atom versus electron transfer. J. Phys. Chem. A 117, 2082-2092. doi: 10.1021/jp3116319

Elhelaly, A. E., AlBasher, G., Alfarraj, S., Almeer, R., Bahbah, E. I., Fouda, M. M. A., et al. (2019). Protective effects of hesperidin and diosmin against acrylamideinduced liver, kidney, and brain oxidative damage in rats. Environ. Sci. Pollut. Res. Int. 26, 35151-35162. doi: 10.1007/s11356-019-06660-3

El-Marasy, S. A., Abdallah, H. M., El-Shenawy, S. M., El-Khatib, A. S., El-Shabrawy, O. A., and Kenawy, S. A. (2014). Anti-depressant effect of hesperidin in diabetic rats. Can. J. Physiol. Pharmacol. 92, 945-952. doi: 10.1139/cjpp-20140281

Erus, G., Battapady, H., Zhang, T., Lovato, J., Miller, M. E., Williamson, J. D., et al. (2015). Spatial patterns of structural brain changes in type 2 diabetic patients and their longitudinal progression with intensive control of blood glucose. Diab. Care 38, 97-104. doi: 10.2337/dc14-1196

Estruel-Amades, S., Massot-Cladera, M., Pérez-Cano, F. J., Franch, À, Castell, M., and Camps-Bossacoma, M. (2019). Hesperidin effects on gut microbiota and gut-associated lymphoid tissue in healthy rats. Nutrients 11:324. doi: 10.3390/ nu11020324

Fidélix, M., Milenkovic, D., Sivieri, K., and Cesar, T. (2020). Microbiota modulation and effects on metabolic biomarkers by orange juice: a controlled clinical trial. Food Funct. 11, 1599-1610. doi: 10.1039/c9fo02623a

Flanagan, E., Lamport, D., Brennan, L., Burnet, P., Calabrese, V., Cunnane, S. C., et al. (2020). Nutrition and the ageing brain: moving towards clinical applications. Ageing Res. Rev. 62:101079. doi: 10.1016/j.arr.2020.101079

Frank, J., Fukagawa, N. K., Bilia, A. R., Johnson, E. J., Kwon, O., Prakash, V., et al. (2020). Terms and nomenclature used for plant-derived components in nutrition and related research: efforts toward harmonization. Nutr. Rev. 78, 451-458. doi: 10.1093/nutrit/nuz081

Fu, H., Liu, L., Tong, Y., Li, Y., Zhang, X., Gao, X., et al. (2019). The antidepressant effects of hesperidin on chronic unpredictable mild stress-induced mice. Eur. J. Pharmacol. 853, 236-246. doi: 10.1016/j.ejphar.2019.03.035

Ghofrani, S., Joghataei, M. T., Mohseni, S., Baluchnejadmojarad, T., Bagheri, M., Khamse, S., et al. (2015). Naringenin improves learning and memory in an Alzheimer's disease rat model: Insights into the underlying mechanisms. Eur. J. Pharmacol. 764, 195-201. doi: 10.1016/j.ejphar.2015.07.001 
Gibon, J., and Barker, P. A. (2017). Neurotrophins and proneurotrophins: focus on synaptic activity and plasticity in the brain. Neuroscientist 23,587-604. doi: $10.1177 / 1073858417697037$

Glass, C. K., Saijo, K., Winner, B., Marchetto, M. C., and Gage, F. H. (2010). Mechanisms underlying inflammation in neurodegeneration. Cell 140, 918934. doi: 10.1016/j.cell.2010.02.016

Goetz, M. E., Judd, S. E., Hartman, T. J., McClellan, W., Anderson, A., and Vaccarino, V. (2016). Flavanone intake is inversely associated with risk of incident ischemic stroke in the reasons for geographic and racial differences in stroke (REGARDS) study. J. Nutr. 146, 2233-2243. doi: 10.3945/jn.116.230185

Gopinath, K., and Sudhandiran, G. (2016). Protective effect of naringin on 3nitropropionic acid-induced neurodegeneration through the modulation of matrix metalloproteinases and glial fibrillary acidic protein. Can. J. Physiol. Pharmacol. 94, 65-71. doi: 10.1139/cjpp-2015-0035

Guirro, M., Gual-Grau, A., Gibert-Ramos, A., Alcaide-Hidalgo, J. M., Canela, N., Arola, L., et al. (2020). Metabolomics elucidates dose-dependent molecular beneficial effects of hesperidin supplementation in rats fed an obesogenic diet. Antioxidants (Basel) 9:79. doi: 10.3390/antiox9010079

Haider, S., Liaquat, L., Ahmad, S., Batool, Z., Siddiqui, R. A., Tabassum, S., et al. (2020). Naringenin protects $\mathrm{AlCl} 3 / \mathrm{D}$-galactose induced neurotoxicity in rat model of $\mathrm{AD}$ via attenuation of acetylcholinesterase levels and inhibition of oxidative stress. PLoS One 15:e0227631. doi: 10.1371/journal.pone.0227631

Hays, R., Henson, P., Wisniewski, H., Hendel, V., Vaidyam, A., and Torous, J. (2019). Assessing cognition outside of the clinic: smartphones and sensors for cognitive assessment across diverse psychiatric disorders. Psychiatr. Clin. North Am. 42, 611-625. doi: 10.1016/j.psc.2019.08.003

Hemanth Kumar, B., Dinesh Kumar, B., and Diwan, P. V. (2017). Hesperidin, a citrus flavonoid, protects against l-methionine-induced hyperhomocysteinemia by abrogation of oxidative stress, endothelial dysfunction and neurotoxicity in Wistar rats. Pharm. Biol. 55, 146-155. doi: 10.1080/13880209.2016.1231695

Heppner, F. L., Ransohoff, R. M., and Becher, B. (2015). Immune attack: the role of inflammation in Alzheimer disease. Nat. Rev. Neurosci. 16, 358-372. doi: $10.1038 / \mathrm{nrn} 3880$

Herrero, M. A.-T., Estrada, C., Maatouk, L., and Vyas, S. (2015). Inflammation in Parkinsonâ $€^{\mathrm{TM}_{\mathrm{S}}}$ disease: role of glucocorticoids. Front. Neuroanatomy 9:32. doi: 10.3389/fnana.2015.00032

Høverstad, T., Carlstedt-Duke, B., Lingaas, E., Norin, E., Saxerholt, H., Steinbakk, M., et al. (1986). Influence of oral intake of seven different antibiotics on faecal short-chain fatty acid excretion in healthy subjects. Scand. J. Gastroenterol. 21, 997-1003. doi: 10.3109/00365528608996411

Hoyles, L., Snelling, T., Umlai, U.-K., Nicholson, J. K., Carding, S. R., Glen, R. C., et al. (2018). Microbiome-host systems interactions: protective effects of propionate upon the blood-brain barrier. Microbiome 6:55. doi: 10.1186/ s40168-018-0439-y

Hritcu, L., Ionita, R., Postu, P. A., Gupta, G. K., Turkez, H., Lima, T. C., et al. (2017). Antidepressant flavonoids and their relationship with oxidative stress. Oxid. Med. Cell Longev. 2017:5762172. doi: 10.1155/2017/57 62172

Hussein, R. M., Mohamed, W. R., and Omar, H. A. (2018). A neuroprotective role of kaempferol against chlorpyrifos-induced oxidative stress and memory deficits in rats via GSK3 $\beta$-Nrf2 signaling pathway. Pestic. Biochem. Physiol. 152, 29-37. doi: 10.1016/j.pestbp.2018.08.008

Iglesias-Carres, L., Mas-Capdevila, A., Bravo, F. I., Aragonès, G., Muguerza, B., and Arola-Arnal, A. (2019). Optimization of a polyphenol extraction method for sweet orange pulp (Citrus sinensis L.) to identify phenolic compounds consumed from sweet oranges. PLoS One 14:e0211267. doi: 10.1371/journal. pone. 0211267

Ishola, I. O., Jacinta, A. A., and Adeyemi, O. O. (2019). Cortico-hippocampal memory enhancing activity of hesperetin on scopolamine-induced amnesia in mice: role of antioxidant defense system, cholinergic neurotransmission and expression of BDNF. Metab. Brain Dis. 34, 979-989. doi: 10.1007/s11011-01900409-0

Jadeja, R. N., and Devkar, R. V. (2014). "Chapter 47 - polyphenols and flavonoids in controlling non-alcoholic steatohepatitis," in Polyphenols in Human Health and Disease, eds R. R. Watson, V. R. Preedy, and S. Zibadi (San Diego, CA: Academic Press), 615-623.

Javed, H., Vaibhav, K., Ahmed, M. E., Khan, A., Tabassum, R., Islam, F., et al. (2015). Effect of hesperidin on neurobehavioral, neuroinflammation, oxidative stress and lipid alteration in intracerebroventricular streptozotocin induced cognitive impairment in mice. J. Neurol. Sci. 348, 51-59. doi: 10.1016/j.jns.2014.10.044

Jellinger, K. A. (2010). Basic mechanisms of neurodegeneration: a critical update. J. Cell. Mol. Med. 14, 457-487. doi: 10.1111/j.1582-4934.2010. 01010.x

Jiao, X., He, P., Li, Y., Fan, Z., Si, M., Xie, Q., et al. (2015). The role of circulating tight junction proteins in evaluating blood brain barrier disruption following intracranial hemorrhage. Dis. Markers 2015:860120. doi: 10.1155/2015/860120

Justin Thenmozhi, A., William Raja, T. R., Manivasagam, T., Janakiraman, U., and Essa, M. M. (2017). Hesperidin ameliorates cognitive dysfunction, oxidative stress and apoptosis against aluminium chloride induced rat model of Alzheimer's disease. Nutr. Neurosci. 20, 360-368. doi: 10.1080/1028415x.2016. 1144846

Kantarci, K., Weigand, S. D., Przybelski, S. A., Preboske, G. M., Pankratz, V. S., Vemuri, P., et al. (2013). MRI and MRS predictors of mild cognitive impairment in a population-based sample. Neurology 81, 126-133. doi: 10.1212/WNL. 0b013e31829a3329

Kay, C. D., Pereira-Caro, G., Ludwig, I. A., Clifford, M. N., and Crozier, A. (2017). Anthocyanins and flavanones are more bioavailable than previously perceived: a review of recent evidence. Annu. Rev. Food Sci. Technol. 8, 155-180. doi: 10.1146/annurev-food-030216-025636

Kean, R. J., Lamport, D. J., Dodd, G. F., Freeman, J. E., Williams, C. M., Ellis, J. A., et al. (2015). Chronic consumption of flavanone-rich orange juice is associated with cognitive benefits: an 8-wk, randomized, double-blind, placebocontrolled trial in healthy older adults. Am. J. Clin. Nutr. 101, 506-514. doi: 10.3945/ajcn.114.088518

Khajevand-Khazaei, M. R., Ziaee, P., Motevalizadeh, S. A., Rohani, M., AfshinMajd, S., Baluchnejadmojarad, T., et al. (2018). Naringenin ameliorates learning and memory impairment following systemic lipopolysaccharide challenge in the rat. Eur. J. Pharmacol. 826, 114-122. doi: 10.1016/j.ejphar.2018. 03.001

Kheradmand, E., Hajizadeh Moghaddam, A., and Zare, M. (2018). Neuroprotective effect of hesperetin and nano-hesperetin on recognition memory impairment and the elevated oxygen stress in rat model of Alzheimer's disease. Biomed. Pharmacother. 97, 1096-1101. doi: 10.1016/j.biopha.2017.11.047

Kiasalari, Z., Khalili, M., Baluchnejadmojarad, T., and Roghani, M. (2016). Protective effect of oral hesperetin against unilateral striatal 6hydroxydopamine damage in the rat. Neurochem. Res. 41, 1065-1072. doi: 10.1007/s11064-015-1796-6

Kipps, C. M., and Hodges, J. R. (2005). Cognitive assessment for clinicians. J. Neurol. Neurosurg. Psychiatry 76(Suppl. 1), i22-i30. doi: 10.1136/jnnp. 2004. 059758

Kosari-Nasab, M., Shokouhi, G., Ghorbanihaghjo, A., Abbasi, M. M., and Salari, A. A. (2018). Hesperidin attenuates depression-related symptoms in mice with mild traumatic brain injury. Life Sci. 213, 198-205. doi: 10.1016/j.lfs.2018. 10.040

Koudoufio, M., Desjardins, Y., Feldman, F., Spahis, S., Delvin, E., and Levy, E. (2020). Insight into polyphenol and gut microbiota crosstalk: are their metabolites the key to understand protective effects against metabolic disorders? Antioxidants (Basel) 9:982. doi: 10.3390/antiox9100982

Kouhestani, S., Jafari, A., and Babaei, P. (2018). Kaempferol attenuates cognitive deficit via regulating oxidative stress and neuroinflammation in an ovariectomized rat model of sporadic dementia. Neural Regen. Res. 13, 18271832. doi: 10.4103/1673-5374.238714

Kreisl, W. C., Kim, M. J., Coughlin, J. M., Henter, I. D., Owen, D. R., and Innis, R. B. (2020). PET imaging of neuroinflammation in neurological disorders. Lancet Neurol. 19, 940-950. doi: 10.1016/s1474-4422(20)30346-X

Krishna Chandran, A. M., Christina, H., Das, S., Mumbrekar, K. D., and Satish Rao, B. S. (2019). Neuroprotective role of naringenin against methylmercury induced cognitive impairment and mitochondrial damage in a mouse model. Environ. Toxicol. Pharmacol. 71:103224. doi: 10.1016/j.etap.2019.103224

Kumar Singh, A., Cabral, C., Kumar, R., Ganguly, R., Kumar Rana, H., Gupta, A., et al. (2019). Beneficial effects of dietary polyphenols on gut microbiota and strategies to improve delivery efficiency. Nutrients 11:2216. doi: 10.3390/ nu11092216

Küster, O. C., Laptinskaya, D., Fissler, P., Schnack, C., Zügel, M., Nold, V., et al. (2017). Novel blood-based biomarkers of cognition, stress, and physical or cognitive training in older adults at risk of dementia: preliminary evidence 
for a role of BDNF, irisin, and the kynurenine pathway. J. Alzheimers Dis. 59, 1097-1111. doi: 10.3233/jad-170447

Kuzu, M., Kandemir, F. M., Yıldırım, S., Çağlayan, C., and Küçükler, S. (2020). Attenuation of sodium arsenite-induced cardiotoxicity and neurotoxicity with the antioxidant, anti-inflammatory, and antiapoptotic effects of hesperidin. Environ. Sci. Pollut. Res. Int. doi: 10.1007/s11356-020-11327-5

Kwon, J. Y., Jung, U. J., Kim, D. W., Kim, S., Moon, G. J., Hong, J., et al. (2018). Beneficial effects of hesperetin in a mouse model of temporal lobe epilepsy. J. Med. Food 21, 1306-1309. doi: 10.1089/jmf.2018.4183

Lamport, D. J., Pal, D., Macready, A. L., Barbosa-Boucas, S., Fletcher, J. M., Williams, C. M., et al. (2016). The effects of flavanone-rich citrus juice on cognitive function and cerebral blood flow: an acute, randomised, placebocontrolled cross-over trial in healthy, young adults. Br. J. Nutr. 116, 2160-2168. doi: $10.1017 /$ s000711451600430x

Lee, B. K., Hyun, S. W., and Jung, Y. S. (2020). Yuzu and hesperidin ameliorate blood-brain barrier disruption during hypoxia via antioxidant activity. Antioxidants (Basel) 9:843. doi: 10.3390/antiox9090843

Li, C., Zug, C., Qu, H., Schluesener, H., and Zhang, Z. (2015). Hesperidin ameliorates behavioral impairments and neuropathology of transgenic APP/PS1 mice. Behav. Brain Res. 281, 32-42. doi: 10.1016/j.bbr.2014.12.012

Li, C. F., Chen, S. M., Chen, X. M., Mu, R. H., Wang, S. S., Geng, D., et al. (2016). ERK-dependent brain-derived neurotrophic factor regulation by hesperidin in mice exposed to chronic mild stress. Brain Res. Bull. 124, 40-47. doi: 10.1016/j. brainresbull.2016.03.016

Li, L., Lyall, G. K., Martinez-Blazquez, J. A., Vallejo, F., Tomas-Barberan, F. A., Birch, K. M., et al. (2020). Blood orange juice consumption increases flowmediated dilation in adults with overweight and obesity: a randomized controlled trial. J. Nutr. 150, 2287-2294. doi: 10.1093/jn/nxaa158

Li, M., Shao, H., Zhang, X., and Qin, B. (2016). Hesperidin alleviates lipopolysaccharide-induced neuroinflammation in mice by promoting the miRNA-132 pathway. Inflammation 39, 1681-1689. doi: 10.1007/s10753-0160402-7

Li, W. H., Cheng, X., Yang, Y. L., Liu, M., Zhang, S. S., Wang, Y. H., et al. (2019). Kaempferol attenuates neuroinflammation and blood brain barrier dysfunction to improve neurological deficits in cerebral ischemia/reperfusion rats. Brain Res. 1722:146361. doi: 10.1016/j.brainres.2019.146361

Liaquat, L., Batool, Z., Sadir, S., Rafiq, S., Shahzad, S., Perveen, T., et al. (2018). Naringenin-induced enhanced antioxidant defence system meliorates cholinergic neurotransmission and consolidates memory in male rats. Life Sci. 194, 213-223. doi: 10.1016/j.lfs.2017.12.034

Lima, A. C. D., Cecatti, C., Fidélix, M. P., Adorno, M. A. T., Sakamoto, I. K., Cesar, T. B., et al. (2019). Effect of daily consumption of orange juice on the levels of blood glucose, lipids, and gut microbiota metabolites: controlled clinical trials. J. Med. Food 22, 202-210. doi: 10.1089/jmf.2018.0080

Lin, L., and Zhang, J. (2017). Role of intestinal microbiota and metabolites on gut homeostasis and human diseases. BMC Immunol. 18:2. doi: 10.1186/s12865016-0187-3

Lou, H., Jing, X., Wei, X., Shi, H., Ren, D., and Zhang, X. (2014). Naringenin protects against 6-OHDA-induced neurotoxicity via activation of the Nrf2/ARE signaling pathway. Neuropharmacology 79, 380-388. doi: 10. 1016/j.neuropharm.2013.11.026

Lu, J. F., Zhu, M. Q., Zhang, H., Liu, H., Xia, B., Wang, Y. L., et al. (2020). Neohesperidin attenuates obesity by altering the composition of the gut microbiota in high-fat diet-fed mice. FASEB J. 34, 12053-12071. doi: 10.1096/fj. 201903102RR

Lv, W. J., Wu, X. L., Chen, W. Q., Li, Y. F., Zhang, G. F., Chao, L. M., et al. (2019). The gut microbiome modulates the changes in liver metabolism and in inflammatory processes in the brain of chronic unpredictable mild stress rats. Oxid. Med. Cell Longev. 2019:7902874. doi: 10.1155/2019/7902874

Magalhães, C. A., Ferreira, C. N., Loures, C. M. G., Fraga, V. G., Chaves, A. C., Oliveira, A. C. R., et al. (2018). Leptin, hsCRP, TNF- $\alpha$ and IL-6 levels from normal aging to dementia: relationship with cognitive and functional status. J. Clin. Neurosci. 56, 150-155. doi: 10.1016/j.jocn.2018.08.027

Mahmoud, A. M., Hernández Bautista, R. J., Sandhu, M. A., and Hussein, O. E. (2019). Beneficial effects of citrus flavonoids on cardiovascular and metabolic health. Oxid. Med. Cell Longev. 2019:5484138. doi: 10.1155/2019/5484138

Manach, C., Morand, C., Gil-Izquierdo, A., Bouteloup-Demange, C., and Rémésy, C. (2003). Bioavailability in humans of the flavanones hesperidin and narirutin after the ingestion of two doses of orange juice. Eur. J. Clin. Nutr. 57, 235-242. doi: $10.1038 /$ sj.ejen. 1601547

Mani, S., Sekar, S., Barathidasan, R., Manivasagam, T., Thenmozhi, A. J., Sevanan, M., et al. (2018). Naringenin decreases $\alpha$-Synuclein expression and neuroinflammation in MPTP-induced Parkinson's disease model in mice. Neurotox Res. 33, 656-670. doi: 10.1007/s12640-018-9869-3

Marchi, N., Cavaglia, M., Fazio, V., Bhudia, S., Hallene, K., and Janigro, D. (2004). Peripheral markers of blood-brain barrier damage. Clin. Chim. Acta 342, 1-12. doi: $10.1016 / j . c c c n .2003 .12 .008$

McPhee, G. M., Downey, L. A., and Stough, C. (2020). Neurotrophins as a reliable biomarker for brain function, structure and cognition: a systematic review and meta-analysis. Neurobiol. Learn. Mem. 175:107298. doi: 10.1016/j.nlm.2020. 107298

Michalska, P., and León, R. (2020). When it comes to an end: oxidative stress crosstalk with protein aggregation and neuroinflammation induce neurodegeneration. Antioxidants (Basel, Switzerland) 9:740. doi: 10.3390/ antiox 9080740

Miners, J. S., Kehoe, P. G., Love, S., Zetterberg, H., and Blennow, K. (2019). CSF evidence of pericyte damage in Alzheimer's disease is associated with markers of blood-brain barrier dysfunction and disease pathology. Alzheimers Res. Ther. 11:81. doi: 10.1186/s13195-019-0534-8

Montagne, A., Nation, D. A., Sagare, A. P., Barisano, G., Sweeney, M. D., Chakhoyan, A., et al. (2020). APOE4 leads to blood-brain barrier dysfunction predicting cognitive decline. Nature 581, 71-76. doi: 10.1038/s41586-0202247-3

Morand, C., Dubray, C., Milenkovic, D., Lioger, D., Martin, J. F., Scalbert, A., et al. (2011). Hesperidin contributes to the vascular protective effects of orange juice: a randomized crossover study in healthy volunteers. Am. J. Clin. Nutr. 93, 73-80. doi: 10.3945/ajen.110.004945

Morand, C., and Tomás-Barberán, F. A. (2019). Contribution of plant food bioactives in promoting health effects of plant foods: why look at interindividual variability? Eur. J. Nutr. 58, 13-19. doi: 10.1007/s00394-019-02096-0

Muhammad, T., Ikram, M., Ullah, R., Rehman, S. U., and Kim, M. O. (2019). Hesperetin, a citrus flavonoid, attenuates LPS-induced neuroinflammation, apoptosis and memory impairments by modulating TLR4/NF-kB signaling. Nutrients 11:648. doi: 10.3390/nu11030648

Musaeus, C. S., Gleerup, H. S., Høgh, P., Waldemar, G., Hasselbalch, S. G., and Simonsen, A. H. (2020). Cerebrospinal fluid/plasma albumin ratio as a biomarker for blood-brain barrier impairment across neurodegenerative dementias. J. Alzheimers Dis. 75, 429-436. doi: 10.3233/jad-200168

Naewla, S., Sirichoat, A., Pannangrong, W., Chaisawang, P., Wigmore, P., and Welbat, J. U. (2019). Hesperidin alleviates methotrexate-induced memory deficits via hippocampal neurogenesis in adult rats. Nutrients 11:936. doi: 10. 3390/nu11040936

Nair, A., and Jacob, S. (2016). A simple practice guide for dose conversion between animals and human. J. Basic Clin. Pharm. 7, 27-31. doi: 10.4103/0976-0105. 177703

Najmanová, I., Vopršalová, M., Saso, L., and Mladěnka, P. (2019). The pharmacokinetics of flavanones. Crit. Rev. Food Sci. Nutr. 60, 3155-3171. doi: 10.1080/10408398.2019.1679085

Nascimento, C. M., Pereira, J. R., de Andrade, L. P., Garuffi, M., Talib, L. L., Forlenza, O. V., et al. (2014). Physical exercise in MCI elderly promotes reduction of pro-inflammatory cytokines and improvements on cognition and BDNF peripheral levels. Curr. Alzheimer Res. 11, 799-805. doi: 10.2174/ 156720501108140910122849

Nation, D. A., Sweeney, M. D., Montagne, A., Sagare, A. P., D’Orazio, L. M., Pachicano, M., et al. (2019). Blood-brain barrier breakdown is an early biomarker of human cognitive dysfunction. Nat. Med. 25, 270-276. doi: 10. 1038/s41591-018-0297-y

Neshatdoust, S., Saunders, C., Castle, S. M., Vauzour, D., Williams, C., Butler, L., et al. (2016). High-flavonoid intake induces cognitive improvements linked to changes in serum brain-derived neurotrophic factor: two randomised, controlled trials. Nutr. Healthy Aging 4, 81-93. doi: 10.3233/nha-1615

Nielsen, I. L. F., Chee, W. S. S., Poulsen, L., Offord-Cavin, E., Rasmussen, S. E., Frederiksen, H., et al. (2006). Bioavailability is improved by enzymatic modification of the citrus flavonoid hesperidin in humans: a randomized, double-blind, crossover trial. J. Nutr. 136, 404-408. doi: 10.1093/jn/136. 2.404 
Noor, M. B. T., Zenia, N. Z., Kaiser, M. S., Mamun, S. A., and Mahmud, M. (2020). Application of deep learning in detecting neurological disorders from magnetic resonance images: a survey on the detection of Alzheimer's disease, Parkinson's disease and schizophrenia. Brain Inform. 7:11. doi: 10.1186/s40708-02000112-2

Nurk, E., Refsum, H., Drevon, C. A., Tell, G. S., Nygaard, H. A., Engedal, K., et al. (2010). Cognitive performance among the elderly in relation to the intake of plant foods. The hordaland health study. Br. J. Nutr. 104, 1190-1201. doi: $10.1017 / \mathrm{s} 0007114510001807$

Okuyama, S., Nakashima, T., Nakamura, K., Shinoka, W., Kotani, M., Sawamoto, A., et al. (2018). Inhibitory effects of auraptene and naringin on astroglial activation, tau hyperphosphorylation, and suppression of neurogenesis in the hippocampus of streptozotocin-induced hyperglycemic mice. Antioxidants (Basel, Switzerland) 7:109. doi: 10.3390/antiox7080109

Palasz, E., Wysocka, A., Gasiorowska, A., Chalimoniuk, M., Niewiadomski, W., and Niewiadomska, G. (2020). BDNF as a promising therapeutic agent in Parkinson's disease. Int. J. Mol. Sci. 21:1170. doi: 10.3390/ijms21031170

Park, M., Choi, J., and Lee, H. J. (2020). Flavonoid-rich orange juice intake and altered gut microbiome in young adults with depressive symptom: a randomized controlled study. Nutrients 12:1815. doi: 10.3390/nu120 61815

Parmenter, B. H., Croft, K. D., Hodgson, J. M., Dalgaard, F., Bondonno, C. P., Lewis, J. R., et al. (2020). An overview and update on the epidemiology of flavonoid intake and cardiovascular disease risk. Food Funct. 11, 6777-6806. doi: $10.1039 / \mathrm{d} 0$ fo01118e

Peng, H. W., Cheng, F. C., Huang, Y. T., Chen, C. F., and Tsai, T. H. (1998). Determination of naringenin and its glucuronide conjugate in rat plasma and brain tissue by high-performance liquid chromatography. J. Chromatogr. B Biomed. Sci. Appl. 714, 369-374. doi: 10.1016/s0378-4347(98)00204-7

Pereira-Caro, G., Polyviou, T., Ludwig, I. A., Nastase, A.-M., Moreno-Rojas, J. M., Garcia, A. L., et al. (2017). Bioavailability of orange juice (poly)phenols: the impact of short-term cessation of training by male endurance athletes. Am. J. Clin. Nutr. 106, 791-800. doi: 10.3945/ajcn.116.149898

Perry, V. H., and Holmes, C. (2014). Microglial priming in neurodegenerative disease. Nat. Rev. Neurol. 10, 217-224. doi: 10.1038/nrneurol.2014.38

Peterson, C. T. (2020). Dysfunction of the microbiota-gut-brain axis in neurodegenerative disease: the promise of therapeutic modulation with prebiotics, medicinal herbs, probiotics, and synbiotics. J. Evid. Based Integr. Med. 25:2515690x20957225. doi: 10.1177/2515690x20957225

Raza, S. S., Khan, M. M., Ahmad, A., Ashafaq, M., Islam, F., Wagner, A. P., et al. (2013). Neuroprotective effect of naringenin is mediated through suppression of NF-кB signaling pathway in experimental stroke. Neuroscience 230, 157-171. doi: 10.1016/j.neuroscience.2012.10.041

Raza, S. S., Khan, M. M., Ahmad, A., Ashafaq, M., Khuwaja, G., Tabassum, R., et al. (2011). Hesperidin ameliorates functional and histological outcome and reduces neuroinflammation in experimental stroke. Brain Res. 1420, 93-105. doi: 10.1016/j.brainres.2011.08.047

Ribeiro-Santos, R., de Campos-Carli, S. M., Ferretjans, R., Teixeira-Carvalho, A., Martins-Filho, O. A., Teixeira, A. L., et al. (2020). The association of cognitive performance and IL-6 levels in schizophrenia is influenced by age and antipsychotic treatment. Nord. J. Psychiatry 74, 187-193. doi: 10.1080/ 08039488.2019.1688389

Rothwell, J. A., Perez-Jimenez, J., Neveu, V., Medina-Remon, A., M'Hiri, N., Garcia-Lobato, P., et al. (2013). Phenol-Explorer 3.0: a major update of the Phenol-Explorer database to incorporate data on the effects of food processing on polyphenol content. Database 2013:bat070. doi: 10.1093/database/bat070

Sachdeva, A. K., Kuhad, A., and Chopra, K. (2014). Naringin ameliorates memory deficits in experimental paradigm of Alzheimer's disease by attenuating mitochondrial dysfunction. Pharmacol. Biochem. Behav. 127, 101-110. doi: 10. 1016/j.pbb.2014.11.002

Schär, M. Y., Curtis, P. J., Hazim, S., Ostertag, L. M., Kay, C. D., Potter, J. F., et al. (2015). Orange juice-derived flavanone and phenolic metabolites do not acutely affect cardiovascular risk biomarkers: a randomized, placebocontrolled, crossover trial in men at moderate risk of cardiovascular disease. Am. J. Clin. Nutr. 101, 931-938. doi: 10.3945/ajcn.114.104364

Scheffers, F. R., Boer, J. M. A., Verschuren, W. M. M., Verheus, M., van der Schouw, Y. T., Sluijs, I., et al. (2018). Pure fruit juice and fruit consumption and the risk of CVD: the european prospective investigation into cancer and nutrition-netherlands (EPIC-NL) study. Br. J. Nutr. 121, 351-359. doi: 10.1017/ s0007114518003380

Shagirtha, K., Bashir, N., and MiltonPrabu, S. (2017). Neuroprotective efficacy of hesperetin against cadmium induced oxidative stress in the brain of rats. Toxicol. Ind. Health 33, 454-468. doi: 10.1177/0748233716665301

Sherwin, E., Dinan, T. G., and Cryan, J. F. (2018). Recent developments in understanding the role of the gut microbiota in brain health and disease. Ann. N. Y. Acad. Sci. 1420, 5-25. doi: 10.1111/nyas.13416

Shivamurthy, V. K., Tahari, A. K., Marcus, C., and Subramaniam, R. M. (2015). Brain FDG PET and the diagnosis of dementia. AJR Am. J. Roentgenol. 204, W76-W85. doi: 10.2214/ajr.13.12363

Silveira, J. Q., Cesar, T. B., Manthey, J. A., Baldwin, E. A., Bai, J., and Raithore, S. (2014). Pharmacokinetics of flavanone glycosides after ingestion of single doses of fresh-squeezed orange juice versus commercially processed orange juice in healthy humans. J. Agric. Food Chem. 62, 12576-12584. doi: 10.1021/jf5038163

Singh, N., Bansal, Y., Bhandari, R., Marwaha, L., Singh, R., Chopra, K., et al. (2017). Naringin reverses neurobehavioral and biochemical alterations in intracerebroventricular collagenase-induced intracerebral hemorrhage in rats. Pharmacology 100, 172-187. doi: 10.1159/000453580

Skillbäck, T., Delsing, L., Synnergren, J., Mattsson, N., Janelidze, S., Nägga, K., et al. (2017). CSF/serum albumin ratio in dementias: a cross-sectional study on 1861 patients. Neurobiol. Aging 59, 1-9. doi: 10.1016/j.neurobiolaging.2017.06.028

Skonieczna-Żydecka, K., Jakubczyk, K., Maciejewska-Markiewicz, D., Janda, K., Kaźmierczak-Siedlecka, K., Kaczmarczyk, M., et al. (2020). Gut biofactoryneurocompetent metabolites within the gastrointestinal tract. A scoping review. Nutrients 12:3369. doi: 10.3390/nu12113369

Sternin, A., Burns, A., and Owen, A. M. (2019). Thirty-five years of computerized cognitive assessment of aging-where are we now? Diagnostics 9:114. doi: 10. 3390/diagnostics9030114

Stevens, Y., Rymenant, E. V., Grootaert, C., Camp, J. V., Possemiers, S., Masclee, A., et al. (2019). The intestinal fate of citrus flavanones and their effects on gastrointestinal health. Nutrients 11:1464. doi: 10.3390/nu11071464

Sugumar, M., Sevanan, M., and Sekar, S. (2019). Neuroprotective effect of naringenin against MPTP-induced oxidative stress. Int. J. Neurosci. 129, 534539. doi: 10.1080/00207454.2018.1545772

Sweeney, M. D., Sagare, A. P., and Zlokovic, B. V. (2018). Blood-brain barrier breakdown in Alzheimer disease and other neurodegenerative disorders. Nat. Rev. Neurol. 14, 133-150. doi: 10.1038/nrneurol.2017.188

Tan, H. E., Sisti, A. C., Jin, H., Vignovich, M., Villavicencio, M., Tsang, K. S., et al. (2020). The gut-brain axis mediates sugar preference. Nature 580, 511-516. doi: 10.1038/s41586-020-2199-7

Tayyab, M., Farheen, S., M, M. M. P., Khanam, N., Mobarak Hossain, M., and Shahi, M. H. (2019). Antidepressant and neuroprotective effects of naringenin via sonic hedgehog-GLI1 cell signaling pathway in a rat model of chronic unpredictable mild stress. Neuromol. Med. 21, 250-261. doi: 10.1007/s12017019-08538-6

Teratani, T., Mikami, Y., Nakamoto, N., Suzuki, T., Harada, Y., Okabayashi, K., et al. (2020). The liver-brain-gut neural arc maintains the Treg cell niche in the gut. Nature 585, 591-596. doi: 10.1038/s41586-020-2425-3

Testai, L., Da Pozzo, E., Piano, I., Pistelli, L., Gargini, C., Breschi, M. C., et al. (2017). The citrus flavanone naringenin produces cardioprotective effects in hearts from 1 year old rat, through activation of mitoBK channels. Front. Pharmacol. 8:71. doi: 10.3389/fphar.2017.00071

Tsai, T. H., and Chen, Y. F. (2000). Determination of unbound hesperetin in rat blood and brain by microdialysis coupled to microbore liquid chromatography. J. Food Drug Anal. 8:1. doi: 10.38212/2224-6614.2814

Vallejo, F., Larrosa, M., Escudero, E., Zafrilla, M. A. P., Cerdaì, B. A., Boza, J., et al. (2010). Concentration and solubility of flavanones in orange beverages affect their bioavailability in humans. J. Agric. Food Chem. 58, 6516-6524. doi: $10.1021 /$ jf100752j

Valls, R. M., Pedret, A., Calderón-Pérez, L., Llauradó, E., Pla-Pagà, L., Companys, J., et al. (2020). Effects of hesperidin in orange juice on blood and pulse pressures in mildly hypertensive individuals: a randomized controlled trial (Citrus study). Eur. J. Nutr. doi: 10.1007/s00394-020-02279-0

Vauzour, D. (2012). Dietary polyphenols as modulators of brain functions: biological actions and molecular mechanisms underpinning their beneficial effects. Oxid. Med. Cell. Longev. 2012, 1-16. doi: 10.1155/2012/ 914273 
Vauzour, D., Camprubi-Robles, M., Miquel-Kergoat, S., Andres-Lacueva, C., Bánáti, D., Barberger-Gateau, P., et al. (2017). Nutrition for the ageing brain: Towards evidence for an optimal diet. Ageing Res. Rev. 35, 222-240. doi: 10. 1016/j.arr.2016.09.010

Wang, D., Liu, L., Zhu, X., Wu, W., and Wang, Y. (2014). Hesperidin alleviates cognitive impairment, mitochondrial dysfunction and oxidative stress in a mouse model of Alzheimer's disease. Cell Mol. Neurobiol. 34, 1209-1221. doi: 10.1007/s10571-014-0098-x

Wang, D., Vogiatzoglou, A., Mulligan, A. A., Lentjes, M. A. H., Luben, R. N., Spencer, J. P. E., et al. (2015a). Flavonoid intake in european adults (18 to 64 years). PLoS One 10:e128132. doi: 10.1371/journal.pone.0128132

Wang, D., Yan, J., Chen, J., Wu, W., Zhu, X., and Wang, Y. (2015b). Naringin improves neuronal insulin signaling, brain mitochondrial function, and cognitive function in high-fat diet-induced obese mice. Cell Mol. Neurobiol. 35, 1061-1071. doi: 10.1007/s10571-015-0201-y

Wang, Y. F., Zheng, L. J., Liu, Y., Ye, Y. B., Luo, S., Lu, G. M., et al. (2019). The gut microbiota-inflammation-brain axis in end-stage renal disease: perspectives from default mode network. Theranostics 9, 8171-8181. doi: 10.7150/thno. 35387

Welbat, J. U., Naewla, S., Pannangrong, W., Sirichoat, A., Aranarochana, A., and Wigmore, P. (2020). Neuroprotective effects of hesperidin against methotrexate-induced changes in neurogenesis and oxidative stress in the adult rat. Biochem. Pharmacol. 178:114083. doi: 10.1016/j.bcp.2020.11 4083

Wium-Andersen, I. K., Rungby, J., Jørgensen, M. B., Sandbæk, A., Osler, M., and Wium-Andersen, M. K. (2019). Risk of dementia and cognitive dysfunction in individuals with diabetes or elevated blood glucose. Epidemiol. Psychiatr. Sci. 29:e43. doi: 10.1017/s2045796019000374

Xiao, J. (2017). Dietary flavonoid aglycones and their glycosides: which show better biological significance? Crit. Rev. Food Sci. Nutr. 57, 1874-1905. doi: 10.1080/ 10408398.2015.1032400

Yang, W., Ma, J., Liu, Z., Lu, Y., Hu, B., and Yu, H. (2014). Effect of naringenin on brain insulin signaling and cognitive functions in ICV-STZ induced dementia model of rats. Neurol. Sci. 35, 741-751. doi: 10.1007/s10072-013-1594-3

Yang, Y., Bai, L., Li, X., Xiong, J., Xu, P., Guo, C., et al. (2014). Transport of active flavonoids, based on cytotoxicity and lipophilicity: an evaluation using the blood-brain barrier cell and Caco-2 cell models. Toxicol. In Vitro 28, 388-396. doi: 10.1016/j.tiv.2013.12.002

Yang, Y. L., Cheng, X., Li, W. H., Liu, M., Wang, Y. H., and Du, G. H. (2019). Kaempferol attenuates LPS-induced striatum injury in mice involving anti-neuroinflammation, maintaining bbb integrity, and down-regulating the HMGB1/TLR4 pathway. Int. J. Mol. Sci. 20:491. doi: 10.3390/ijms20030491

Yassine, H. N., Croteau, E., Rawat, V., Hibbeln, J. R., Rapoport, S. I., Cunnane, S. C., et al. (2017). DHA brain uptake and APOE4 status: a PET study with [1-(11)C]-DHA. Alzheimers Res. Ther. 9:23. doi: 10.1186/s13195-0170250-1

Yi, L. T., Liu, B. B., Li, J., Luo, L., Liu, Q., Geng, D., et al. (2014). BDNF signaling is necessary for the antidepressant-like effect of naringenin. Prog. Neuropsychopharmacol. Biol. Psychiatry 48, 135-141. doi: 10.1016/j.pnpbp. 2013.10.002

Youdim, K. A., Dobbie, M. S., Kuhnle, G., Proteggente, A. R., Abbott, N. J., and Rice-Evans, C. (2003). Interaction between flavonoids and the blood-brain barrier: in vitro studies. J. Neurochem. 85, 180-192. doi: 10.1046/j.1471-4159. 2003.01652.x

Youdim, K. A., Qaiser, M. Z., Begley, D. J., Rice-Evans, C. A., and Abbott, N. J. (2004). Flavonoid permeability across an in situ model of the blood-brain barrier. Free Radical Biol. Med. 36, 592-604. doi: 10.1016/j.freeradbiomed.2003. 11.023

Yu, Y., Tang, D., and Kang, R. (2015). Oxidative stress-mediated HMGB1 biology. Front. Physiol. 6:93.

Zamora-Ros, R., Knaze, V., Rothwell, J. A., Hémon, B., Moskal, A., Overvad, K., et al. (2015). Dietary polyphenol intake in Europe: the European prospective investigation into cancer and nutrition (EPIC) study. Eur. J. Nutr. 55, 13591375. doi: 10.1007/s00394-015-0950-X

Zhang, S., Tomata, Y., Sugiyama, K., Sugawara, Y., and Tsuji, I. (2017). Citrus consumption and incident dementia in elderly Japanese: the Ohsaki Cohort 2006 Study. Br. J. Nutr. 117, 1174-1180. doi: 10.1017/s000711451700109x

Zhou, T., Liu, L., Wang, Q., and Gao, Y. (2020). Naringenin alleviates cognition deficits in high-fat diet-fed SAMP8 mice. J. Food Biochem. 44:e13375. doi: $10.1111 /$ jfbc. 13375

Zhu, J. C., Si, M. Y., Li, Y. Z., Chen, H. Z., Fan, Z. C., Xie, Q. D., et al. (2017). Circulating tight junction proteins mirror blood-brain barrier integrity in leukaemia central nervous system metastasis. Hematol. Oncol. 35, 365-373. doi: 10.1002/hon.2289

Zhu, X., Liu, H., Liu, Y., Chen, Y., Liu, Y., and Yin, X. (2020). The antidepressantlike effects of hesperidin in streptozotocin-induced diabetic rats by activating Nrf2/ARE/Glyoxalase 1 pathway. Front. Pharmacol. 11:1325. doi: 10.3389/ fphar.2020.01325

Zilberter, Y., and Zilberter, M. (2017). The vicious circle of hypometabolism in neurodegenerative diseases: ways and mechanisms of metabolic correction. J. Neurosci. Res. 95, 2217-2235. doi: 10.1002/jnr.24064

Zurbau, A., Au-Yeung, F., Blanco Mejia, S., Khan, T. A., Vuksan, V., Jovanovski, E., et al. (2020). Relation of different fruit and vegetable sources with incident cardiovascular outcomes: a systematic review and meta-analysis of prospective cohort studies. J. Am. Heart Assoc. 9:e017728. doi: 10.1161/jaha.120. 017728

Conflict of Interest: MP and DV received unrestricted honorariums from the Fruit Juice Science Centre. The article reflects the views of the authors alone, and the funding source had no role in the preparation or submission of the manuscript.

The remaining authors declare that the research was conducted in the absence of any commercial or financial relationships that could be construed as a potential conflict of interest.

The handling editor declared a past co-authorship with one of the authors DV.

Copyright (c) 2021 Pontifex, Malik, Connell, Müller and Vauzour. This is an openaccess article distributed under the terms of the Creative Commons Attribution License (CC BY). The use, distribution or reproduction in other forums is permitted, provided the original author(s) and the copyright owner(s) are credited and that the original publication in this journal is cited, in accordance with accepted academic practice. No use, distribution or reproduction is permitted which does not comply with these terms. 